\title{
MRI As A Diagnostic Tool In Evaluating Internal Derangements of Knee As Compared to Arthroscopy
}

\author{
Authors \\ Dr Naresh Nikhil. B.L ${ }^{1}$, Dr Harshita. H. $S^{2}$ \\ ${ }^{1}$ Senior Resident, Dept of Radio-Diagnosis, Bangalore Medical College and Victoria Hospital, Bangalore \\ ${ }^{2}$ Junior Resident, Dept of Radio-Diagnosis, Rajajajeswari Medical College and Hospital, Bangalore
}

\section{INTRODUCTION}

Knee joint is the largest and the most complex joint in the body and also the most frequently injured joint due to its anatomy. The stability of the joint is highly dependent on its supporting ligamentous structures. Injuries of the ligaments and the menisci are extremely common. Injury to the knee joint is a significant cause of morbidity in both young and old individuals. As our institution is a tertiary centre, the cases from the primary centers are referred. An accurate diagnosis regarding the involvement of structures and the extent of injuries is essential for early operative as well as non-operative treatment. This requires an accurate clinical history, a thorough physical examination and complementary diagnostic tools.

The most widely used diagnostic modalitiy to assess the joint injury is arthroscopy and MRI.

Arthroscopy is gold standard to diagnose the intra-articular knee pathology but it is an invasive procedure and can cause complications, So. Magnetic resonance imaging (MRI)is increasingly being used for diagnosis over the past decade ${ }^{(1,2 .)}$. MRI has now been accepted as the best imaging modality for non-invasive evaluation of knee injuries ${ }^{(3)}$. Since its introduction for clinical use in the mid-1980s, the role of MRI in the diagnosis of knee lesions has been well established. The ability of MRI to predict intra-articular knee pathology has been compared with findings of arthroscopic results ${ }^{[1,3-5]}$.The accuracy and sensitivity and specificity vary in different studies. This variety is due to changes in MRI technology and difference in imaging sequences, expertise of the radiologist, diagnostic criteria and the kind of knee injury. As a result of these variability, this study was decided to determine the correlation between arthroscopy and MRI findings of knee joint injuries.

\section{AIMS AND OBJECTIVES OF STUDY}

- To study and characterize the MRI findings of the knee joint in patients referred to MRI with a history of trauma from orthopaedic OPD.

- Grading of the various ligamentous and meniscal injuries on the basis of laid down criteria.

- $\quad$ To correlate the MRI findings with Arthroscopic findings.

\section{REVIEW OF LITERATURE}

In a study done by David A.Turner et.al ${ }^{11}$ in 1985, they concluded that MR imaging may be useful in detecting acute injury of ligaments of the knee by comparing 13 cases with the arthroscopy and/or arthrotomy . 
In a study done by Lee JK et.al ${ }^{12}$ in 1988 , they compared the MR with the findings of two commonly applied clinical tests of ACL instability: the Lachman test and the anterior drawer test and arthroscopy got the following results. The sensitivity of MR imaging was 94\% (17 of 18), compared with 78\% (14 of 18) for the anterior drawer test and 89\% (16 of 18) for the Lachman test. The specificity was $100 \%$ for all three. Three MR criteria were applied: irregularity or a wavy contour of the anterior margin of the ligament, high-signal-intensity change within the substance of the ligament on T2-weighted images, and discontinuity of that substance. The study concluded that the sagittal T2-weighted image was especially helpful, producing an "arthrographic" effect, in which the anterior margin of the ACL is outlined by high-signal-intensity joint fluid. By demonstrating ACL and other extrameniscal lesions, MR imaging may help clarify the mechanisms of knee injury.

In a study done by Barronian $\mathrm{AD}^{13}$ in 1989 , he compared the MRI findings with arthroscopic findings of meniscal, ligamentous, and articular cartilaginous pathology using a double-blind prospective protocol. The overall accuracy for meniscal tears was $78 \%$, with a sensitivity of $88 \%$ and a specificity of $72 \%$; for cruciate ligaments, MRI was $82 \%$ accurate, with a sensitivity of $67 \%$ [anterior cruciate ligament (ACL) only] and a specificity of $86 \%$. In select clinical situations, MRI of the knee is a useful diagnostic tool.

In a study done by Heron CW et.al ${ }^{14}$ in 1992, they concluded that Three-dimensional GRE MR imaging enables accurate assessment of the articular cartilage of the knee. The evaluation of meniscal tears and the cruciate ligaments has a high negative predictive value. The sensitivity of MR imaging in diagnosis of meniscal tears seen at arthroscopy was $97 \%$ and the specificity, $94 \%$. For complete tears of the anterior cruciate ligament, the sensitivity was $92 \%$ and specificity, $96 \%$. In the posterior cruciate ligament, both the sensitivity and specificity were $100 \%$. Good correlation existed between findings at MR imaging and those at arthroscopy in assessment of focal thinning and full-thickness loss of hyaline cartilage, but arthroscopy enabled superior visualization of minor fissuring.

In a study done by Tung $\mathrm{GA}^{15}$ in 1993, the study showed that primary signs of ACL tear (ie, abnormal ACL morphologic features or signal intensity) had respective sensitivity and specificity values of $96 \%$ (48 of 50 examinations) and $94 \%$ (50 of 53) on sagittal images and $92 \%$ (46 of 50) and 83\% (43 of 52) on coronal images. As a secondary sign of ACL tear, bone bruise involving the lateral compartment of the knee was found in 40\% (20 of 50) of cases of ACL tear and in 4\% (2 of 53) of cases of normal ACL. The mean curvature of the posterior cruciate ligament was increased $(0.40$ vs $0.27 ; \mathrm{P}<.0001)$ in cases of ACL tear. An abnormal appearance of the ACL on sagittal images remains the single most sensitive and specific sign of ACL tear.

In a study done by Raunest $\mathrm{J}$ et.al ${ }^{16}$ in 1994 , the correlation between MRI findings and arthroscopic diagnoses with a histologic grading model got the following results. In $82.8 \%$ of the results, grading based on MRI studies corresponded with the histologic grading classification. In 12 instances a meniscal degeneration verified at light microscopy was not detected at MRI, whereas in 15 cases tomography yielded a false-positive result. The overall accuracy was calculated to be 0.93 with a specificity of 0.79 and a sensitivity of 0.96 . Concerning the evaluation of meniscal degenerations, MRI provides a positive predictive value of 0.95 and a negative predictive value of 0.82 . Compared with the diagnostic specificity of the anterior and posterior zones, that of the intermediate segment of the meniscus is significantly reduced $(\mathrm{p}<0.001)$. At arthroscopy, meniscal degenerations were diagnosed with an overall accuracy of $38.8 \%$, a sensitivity of $27.5 \%$, and a specificity of $75.5 \%$. In 80 cases of grade 3 abnormalities, five false-negative diagnoses were made initially. These results suggest that MRI offers a valuable diagnostic potential providing reliable information about the internal consistency of the meniscus complementary to diagnostic arthroscopy. .

In a study done by Mackenzie R et.al ${ }^{17}$ in 1996 it was concluded that Magnetic resonance imaging significantly influences clinicians' diagnoses and management plans. These patients, examined by MRI, also recorded an improvement in health related quality of life. Study in a 332 patients. MRI led to previously unsuspected diagnosis in 69 of 269 patients with available data. When MRI confirmed the clinical diagnosis, significant improvements in clinicians' diagnostic confidence were found (P $<0.01$ for medical meniscus, $\mathrm{P}<0.05$ lateral meniscus, $\mathrm{P}<0.05$ anterior cruciate). MRI led to a change in management in $180(63 \%)$ of 288 patients (where data available). There was a significant shift away from surgical management after MRI (P < 0.01$)$. SF-36 results were available in 206 patients. There was a significant improvement over time in five of the eight SF-36 scales (four at $\mathrm{P}<$ 0.001 , one at $\mathrm{P}<0.01)$.

In a study done by Carmichael IW et.al ${ }^{18}$ in 1997 found that MRI is a reliable and cheaper alternative to 'diagnostic arthroscopy'. We consider that patients with definite clinical signs merit an early 'therapeutic arthroscopy', but that all other knees should be investigated by MRI. This policy spares patients from unnecessary and expensive surgery. Arthroscopy for diagnostic purposes should be used only with a specific purpose. Modern MRI can and should replace "having a look" with the use of MRI and arthroscopy to diagnose problems of the knee 324 arthroscopies and $66 \mathrm{MR}$ scans by four orthopaedic consultants. 
In a study done by Rappeport $\mathrm{ED}^{19}$ in 1997 it was concluded that low-field MRI can be used as a first-line diagnostic examination in patients with suspected meniscus or cruciate ligament injuries and thus a substantial number of negative diagnostic arthroscopies can be avoided. The accuracy rates of MRI for evaluating the medial meniscus, lateral meniscus and anterior cruciate ligament were $77 \%, 91 \%$ and $96 \%$, respectively, when arthroscopy was considered the "gold standard". When MRI was considered the standard, the figures for arthroscopy were $74 \%, 91 \%$ and $96 \%$. MRI found the indication for treatment in 18 of 21 patients who were treated at the arthroscopy. In 17 patients, neither MRI nor arthroscopy detected any lesion. In the remaining 9 patients, MRI demonstrated a lesion, but no lesion was found at the subsequent arthroscopy.

In a study done by Cheung $\mathrm{LP}^{20}$ in 1997 it was concluded that the Fast SE imaging of the knee can be an alternative to conventional SE imaging for the detection of meniscal tears. The sensitivity and specificity for 143 confirmed tears of the medial meniscus were $89 \%$ and $84 \%$, respectively, while the values for 96 confirmed tears of the lateral meniscus were $72 \%$ and $93 \%$. These values are within the ranges of values recently reported for imaging strategies relying predominantly on conventional SE sequences.

In a study done by Steven Schultz et.al ${ }^{21}$ in 1997 it was concluded that MRI can be a valuable adjunct in medical diagnoses, and the use of MRI will continue to evolve as an important part of surgical planning. This study showed good overall validity with the use of mid-field MRI to diagnose knee structures. Accuracy was highest in the detection of ACL tears (96\%) and lowest in finding medial meniscal tears (89\%). Sensitivity was lower in the lateral meniscus compared to the medial meniscus $(69 \%$ vs $94 \%$, respectively), and lower specificity occurred with the medial meniscus compared to the lateral meniscus (88\% vs $94 \%$, respectively).

In a study done by Maurer EJ ${ }^{22}$ in 1997 study it was concluded that MR imaging affects the diagnosis and management of acute knee injury by decreasing the number of arthroscopic procedures, improving clinician diagnostic certainty, and assisting in management decisions The increase in diagnostic certainty was greatest for medial meniscal injuries (30\%), followed by lateral meniscal injuries (21\%). The proposed management changed in 41 patients, resulting in significantly fewer arthroscopic procedures $(\mathrm{P}<.01)$. The post-MR imaging management plans included 37\% (27 of 73) fewer arthroscopic procedures.

In a study done by Bui-Mansfield $\mathrm{LT}^{23}$ in 1997, the study indicated that MR imaging obtained before arthroscopy of the knee can produce a savings of as much as $\$ 680$ per MR imaging examination performed on the knee. The respective sensitivities and specificities of MR imaging of the knee were $100 \%$ and $90 \%$ for revealing anterior cruciate ligament interruption, $100 \%$ and $84 \%$ for revealing posterior cruciate ligament tears, $90 \%$ and $97 \%$ for revealing medial meniscal tears, $60 \%$ and $100 \%$ for revealing lateral meniscal tears, and $94 \%$ and $93 \%$ for revealing composite injury (one or more of these abnormalities). With MR imaging examinations obtained before surgery, we found 21 (42\%) of 50 arthroscopies to be unnecessary.

In a study done by Stanitski CL ${ }^{24}$ in 1998 it was concluded that a highly positive correlation (78.5\%) was found between clinical and arthroscopic findings. A highly negative correlation was found between arthroscopic and magnetic resonance imaging findings (78.5\%) and between clinical and magnetic resonance imaging findings (75\%). In this series, accuracy, positive predictive value, negative predictive value, sensitivity, and specificity data were much more favorable from clinical examination than from magnetic resonance imaging. Overall, magnetic resonance imaging diagnoses added little guidance to patient management and at times provided spurious information.

In a study done by Kreitner KF et.al ${ }^{25}$ in 1998 it was concluded that discrepant findings between MRI and arthroscopy may be also due to an insufficient arthroscopic evaluation in clinical routine. The close cooperation between surgeons and radiologists improves the understanding of the methods of each other. The diagnostic accuracies for injuries of the medial and lateral meniscus and the ACL were $92.4 \%, 92.4 \%$, and 94.1\%, respectively. For the menisci, causes for discrepancies in findings ( $\mathrm{n}=31$ ) were: over interpretation of central signal intensities with contact to the meniscal surface but without disturbance of the meniscal contour as a tear $(n=12)$, insufficient arthroscopic evaluation of the knee joint $(n=11)$, overlooked tears on MR imaging $(n=6)$, misinterpretation of normal anatomic structures $(n=1)$, "magic angle" phenomenon $(n=1)$, and missed tears at MRI $(n=1)$. Causes for discrepancies for the ACL $(n=18)$ were: 1$)$ nearly complete versus complete rupture either at MRI or arthroscopy and vice versa $(n=9), 2)$ insufficient arthroscopic evaluation $(n=6)$, 3)insufficient MRI technique $(n=2)$, and 4)overlooked tear on MR imaging $(\mathrm{n}=1)$.

In a study done by Munk Bet.al ${ }^{26}$ in 1998 , in meniscal tears, the accuracy and positive predictive value of MRI was found to be nearly twice that of clinical examination. The sensitivity, specificity, and negative predictive value of MRI were comparable to the figures found in other studies. We recommend MRI as a clarifying diagnostic tool when a clinical examination indicates a lesion 
of the meniscus. In our study, the clinical relevance of MRI in anterior cruciate ligament lesions and especially in cartilage lesions was more doubtful. The combination of clinical and MRI findings would reduce the number of blank arthroscopies to 5\%. MRI is a valuable diagnostic tool in planning the type of anesthesia and treatment, and could significantly reduce the need for a second arthroscopy.

In a study done by Kwanseop Lee et. $\mathrm{al}^{67}$ in 1999 , Anterior Cruciate Ligament Tears: MR Imaging-based diagnosis in a Pediatric Population study concluded that Primary and secondary findings of ACL tears in young patients have high specificity and are useful for diagnosis. Overall sensitivity and specificity of MR imaging in detecting ACL tears were $95 \%$ and $88 \%$, respectively. Sensitivities of the primary findings were $94 \%$ for abnormal Blumensaat angle; $79 \%$, abnormal signal intensity; and $21 \%$, discontinuity. The specificity of all primary findings was $88 \%$ or greater. The sensitivity and specificity of the secondary findings, respectively, were $68 \%$ and $88 \%$ for bone bruise; $63 \%$ and $92 \%$, anterior tibial displacement; $42 \%$ and $96 \%$, uncovered posterior horn of lateral meniscus; $68 \%$ and $92 \%$, positive posterior cruciate line; and $74 \%$ and $71 \%$, abnormal posterior cruciate angle. Fifteen $(79 \%)$ patients had meniscal tears, and five $(26 \%)$ had collateral ligament injuries.

In a study done by Munshi $\mathrm{M}$ et.al ${ }^{27}$ in 2000 , it was concluded that Equivocal clinical findings in patients with acute knee injury should lead to use of MRI in an appropriate clinical setting. This study supported the use of early MRI to guide further surgical management. The respective sensitivity and specificity for MRI of the knee were $90 \%$ (18/20) and 67\% (2/3) for detecting any anterior cruciate ligament injury, 50\% (1/2) and 86\% (18/21) for detecting medial meniscal tears, and 88\% (7/8) and 73\% (11/15) for detecting lateral meniscal tears. MRI also identified injuries that could not be assessed on arthroscopy, including 14 bone bruises, five posterior cruciate ligament tears, nine medial collateral ligament tears, and one lateral collateral ligament tear. The detection of composite injury requiring surgical intervention yielded a sensitivity of 100\% (16/16) and a specificity of 71\% (5/7). Prospective use of MRI evaluation of the knee could have prevented 22\% (5/23) of diagnostic arthroscopic procedures.

In a syudy done by Zairul-Nizam $\mathrm{ZF}^{28}$ in 2000 it was concluded that MRI examination is an effective first-line investigation for patients with suspected internal knee derangement, especially different surgical approaches exist for many cases. This unit presents the standard protocol for imaging injured knees in a clinical setting.

In a study done by DS Shetty et.al ${ }^{29}$ in 2002 it was concluded that MR imaging of knee is an excellent modality that detects lesions not evident on arthroscopy and helps in planning the treatment of meniscal and ligament injuries. It demonstrates extent of marrow and soft tissue involvement of tumors with great degree of accuracy. Hyperintensity, discontinuity and nonvisualisation were primary signs of ACL tear. Secondary signs like Posterior cruciate ligament (PCL) buckling, PCL index of greater than 0.5 and uncovered lateral meniscus (LM) were also noted in certain areas. Meniscal tears seen commonly are grade III in medial meniscus and Grade lin lateral meniscus. Cases of meniscal cyst, synovial chondromatosis, quadriceps tendon tear, osteochondritis dissecans are clearly demonstrated on MR scans. The exact extent of the tumor in the marrow and soft tissues and involvement of joint space was demonstrated with great degree of accuracy.

In a study of Effectiveness of MR imaging in selection of patients for arthroscopy of the knee Vincken PW et.al ${ }^{30}$ in 2002 concluded MR imaging is an effective tool in the selection of patients for arthroscopy from among a general population. Sensitivity and specificity corrected for randomization were $87.3 \%$ and $88.4 \%$. In a study done for 200 patients, Sensitivity and specificity corrected for randomization, respectively were $84.1 \%$ and $94.2 \%$ for the diagnosis of medial meniscal tears and $69.5 \%$ and $94.5 \%$ for the diagnosis of lateral meniscal tears.

It a study done by Major $\mathrm{NM}^{31}$ in 2003 it was suggested that MR imaging of the knee in adolescents is sensitive, specific, and accurate. The sensitivity and specificity values for MR imaging of the menisci and cruciate ligaments in adolescents were as follows: medial meniscus, $92 \%$ sensitivity and $87 \%$ specificity; lateral meniscus, $93 \%$ sensitivity and $95 \%$ specificity; anterior cruciate ligament, $100 \%$ sensitivity and $100 \%$ specificity; and posterior cruciate ligament, $0 \%$ sensitivity and $100 \%$ specificity.

In a study done by Stirling Bryan ${ }^{32}$ et.al it was concluded that the use of MRI in patients with chronic knee problems, in whom surgery was being considered, did not increase costs overall, was not associated with worse outcomes, and avoided surgery in a significant proportion of patients in 2004 .

In a study done by J.P Singh ${ }^{6}$ in 2004 it was concluded that MRI is a very useful non-invasive diagnostic modality having high sensitivity, specificity and accuracy in the identification of meniscal and cruciate ligament injuries. MRI should be done in every patient of suspected ligamentous injury, to be posted for arthroscopy thus preventing unwanted arthroscopies. Sensitivity, Specificity and Accuracy of MRI in detecting meniscal and cruciate ligamentous injuries as compared to arthroscopy is as 
follows: Medial Meniscus (MM): 96.5 percent, 98.28 percent, 97.69 percent; for Lateral Meniscus (LMP:87 percent, 99.29 percent, 97.11 percent, Anterior Cruciate Ligament (ACL):98.72 percent, 98.94 percent, 98.84 percent; and for Posterior Cruciate Ligament (PCL): 98.72 percent, 98.94 percent, 98.84 percent. Most commonly Medial Meniscus (57 patients:32\%) and anterior cruciate ligament (78 patients:45 \%) injury was seen involving the posterior horn of Medial Meniscus (45 patients:79 \%), and midsubstance Anterior Cruciate Ligament (53 patients:68 \%) respectively.

In a study done by Chang CY et.al ${ }^{33}$ in 2004 it was shown that the sensitivity and specificity for meniscal tears were $92 \%$ and $87 \%$, respectively. Type VI meniscal tear was the most common type, especially in displaced meniscal tear. MR is a reliable diagnostic tool for meniscal tears and associated cruciate ligament injury.

In a study done by Thomas Magee et.al ${ }^{34}$ in 2004 it was concluded that coronal MR images of the knee allowed better detection and characterization of some meniscal tears than sagittal images alone. Radial meniscal tears, bucket handle tears and horizontal tears in the body of the meniscus may be difficult to characterize in the sagittal plane alone. Use of coronal T1-weighted images, rather than coronal T2-wweighted or sagittal proton density images alone, allows accurate characterization of some additional marrow lesions. The addition of a t1-weighted sequence in the coronal plane adds only $1 \mathrm{~min} 30 \mathrm{sec}$ to the scanning time.

MRI examinations that demonstrate no significant effusions are most often free of internal derangement, and those which show significant effusions exhibit internal derangement. An anteroposterior measurement of $10 \mathrm{~mm}$ or less in the lateral aspect of the suprapatellar pouch is a reasonable threshold value for distinguishing a physiologic from a pathologic amount of joint fluid was concluded by Kolman B H et.al ${ }^{35}$ in 2004 study.

In a study done by Kocabey $\mathrm{Y}$ et.al ${ }^{36}$ in 2004 it was shown that no statistical difference between MRI or clinical examination in diagnosing medial or lateral meniscal tears or ACL tears $(\mathrm{P}>.05)$. The accuracy of the clinical examination and MRI evaluation was equal for diagnosing meniscal tears and ACL ruptures and concluded that a well-trained qualified surgeon can safely rely on clinical examination for diagnosing meniscal and ACL injuries. Clinical examination is at least as accurate as MRI in the skilled orthopaedic surgeon's hand. MRI should be reserved for more complicated and confusing cases. The routine ordering of an MRI scan of the knee before examination by a well-trained orthopaedic surgeon is not recommended.

In a study done by Jee $\mathrm{WH}^{37}$ in 2004 it was shown that in presence of acute anterior cruciate ligament tears, MRI imaging has relatively low sensitivity for detecting meniscal tears due to missed tears in the lateral meniscus With MR imaging the sensitivity, specificity and accuracy for diagnosing meniscal tears in the presence of acute anterior cruciate ligament tears were $71 \%, 93 \%$, and $88 \%$; for the lateral meniscal tears were $57 \%, 100 \%$ and $85 \%$; and for the medial meniscal tears were $100 \%, 88 \%, 90 \%$. All false negative cases $(n=6)$ involved the posterior horn of the lateral meniscus.

In a study done by Luhmann $\mathrm{SJ}^{38}$ in 2005 , it was shown that Integration of patient information with an orthopaedic surgeon's review of the magnetic resonance imaging scan of the knee in children and adolescent patients improves the identification of pathological disorders in four of the six categories evaluated. This study questions the necessity for and appropriateness of a routine interpretation of a magnetic resonance imaging scan of the knee in children and adolescents by a radiologist. Relative to operative findings, kappa values for the formal interpretations of the magnetic resonance imaging scans by a radiologist were 0.78 for an anterior cruciate ligament tear, 0.76 for a medial meniscal tear, 0.71 for a lateral meniscal tear, 0.70 for osteochondritis dissecans, 0.46 for discoid lateral meniscus, and 0.65 for osteochondral fracture. Relative to operative findings, kappa values for the preoperative diagnoses by the surgeon were 1.00 for an anterior cruciate ligament tear, 0.90 for a medial meniscal tear, 0.92 for a lateral meniscal tear, 0.93 for osteochondritis dissecans, 1.00 for discoid lateral meniscus, and 0.90 for osteochondral fracture. The preoperative diagnosis by the surgeon was better $(\mathrm{p}<0.05)$ than the formal interpretation of the magnetic resonance imaging scans by the radiologist with respect to an anterior cruciate ligament tear, lateral meniscal tear, osteochondritis dissecans, and discoid lateral meniscus.

In a study done by Esmaili $\mathrm{Jah}^{39}$ AA et.al in 2005 an excellent correlation between MRI and clinical findings were found. However, when MRI is normal, high clinical suspicion and a skilled clinical examination are more reliable. Clinical examination was accurate in $91.4 \%$, and MRI in $88.5 \%$ of anterior cruciate ligament injuries. For posterior cruciate ligament injuries, clinical accuracy was $100 \%$ and MRI $94.6 \%$. Clinical examination was accurate in $96.9 \%$ and MRI in $85.9 \%$ of medial meniscal injuries. For lateral meniscus injuries, clinical accuracy was $85.4 \%$ and MRI 73.8\%. MRI findings showed the lowest correlation with arthroscopic findings in lateral meniscus injuries $(r=0 / 47)$. 
In a study done by Carlos Eduardo Sanches $\mathrm{Vaz}^{40}$ in 2005 , concluded that Magnetic resonance imaging is a satisfactory diagnostic tool for evaluating meniscal and ligamentous lesions of the knee, but it is unable to clearly identify articular cartilage lesions. The study resulted as follows : medial meniscus: sensitivity $97.5 \%$, specificity $92.9 \%$, positive predictive value $93.9 \%$, positive negative value $97 \%$, likelihood positive ratio 13.7 , likelihood negative ratio 0.02 , and accuracy $95.3 \%$; lateral meniscus: sensitivity $91.9 \%$, specificity $93.6 \%$, positive predictive value $92.7 \%$, positive negative value $92.9 \%$, likelihood positive ratio 14.3, likelihood negative ratio 0.08, and accuracy 93.6\%; anterior cruciate ligament: sensitivity $99.0 \%$, specificity $95.9 \%$, positive predictive value $91.9 \%$, positive negative value $99.5 \%$, likelihood positive ratio 21.5 , likelihood negative ratio 0.01 , and accuracy 96.6\%; posterior cruciate ligament: sensitivity $100 \%$, specificity $99 \%$, positive predictive value $80.0 \%$, positive negative value $100 \%$, likelihood positive ratio 100 , likelihood negative ratio 0.01 , and accuracy $99.6 \%$; articular cartilage: sensitivity $76.1 \%$, specificity $94.9 \%$, positive predictive value $94.7 \%$, positive negative value $76.9 \%$, likelihood positive ratio 14.9 , likelihood negative ratio 0.25 , and accuracy $84.6 \%$.

In a study done by Winters $\mathrm{K}$ et.al ${ }^{41}$ in 2005 , the reliability of magnetic resonance imaging of the traumatic knee as determined by arthroscopy study results are diagnostic accuracy of $90 \%$ for the medial meniscus, $82 \%$ for the lateral meniscus, $94 \%$ for the anterior cruciate ligament, and $96 \%$ for the posterior cruciate ligament. The sensitivity was $87 \%$ for the medial meniscus, only $46 \%$ for the lateral meniscus, $92 \%$ for the anterior cruciate, and $80 \%$ for the posterior cruciate. The specificity was relatively high at $92 \%, 91 \%, 94 \%$, and $97 \%$, respectively.

In a study done by Mahmoud Karimi-Mobarake et.al ${ }^{42}$ in 2005, it was shown that the accuracy, sensitivity, specificity, positive and negative predictive values were respectively $72.2,100,61.5,50$ and 100\% for medial meniscus tear and 88.8, 80,92.3,80 and $92.3 \%$ for lateral meniscus tear. And $100 \%$ for anterior cruciate ligament tear.MRI is a safe and usefull adjunct to the clinical examination of the injured knee and an aid to efficient preoperative planning.

In a study done by Patrice W. J. Vincken et.al ${ }^{43}$ in 2006, it was found that the MR imaging can be used without additional costs or disadvantageous effects on function to obviate arthroscopy in patients with nonacute knee symptoms who are highly suspected of having intraarticular knee abnormality.

In a study done by R. Richard Ramnat ${ }^{44}$ in 2006, it was concluded that 3-T MRI using fast spin-echo sequences is highly accurate in the detection of medial and lateral meniscal tears of the knee.

In a study done by Bridgman $\mathrm{S}$ et.al ${ }^{45}$ in 2007, it was found that there was no effect of MRI on the decision to perform arthroscopy or patient outcome. Performing MRI in patients already on the waiting list for arthroscopy may not be effective in reducing utilization of surgery. Surgeons changed both their diagnosis and management plan in $47 \%$ of patients in the intervention group compared with $1 \%$ in the control group, with no difference between groups in the proportion of patients who underwent an arthroscopy. In the intervention group 7 of 125 patients $(5.6 \%)$ did not have an arthroscopy compared with 8 of 127 patients $(6.3 \%)$ in the control group. In one instance a surgeon decided against arthroscopy based on the MRI report. There was no significant difference between groups in other outcome measures.

In a study done by B. R. Mohan et.al ${ }^{46}$ in 2007 showed an accuracy similar to most of the MRI studies on meniscal tears and concluded that physical examination is a useful and important diagnostic technique and is as reliable as MRI to diagnose meniscal tears and recommend the use of MRI for more doubtful, difficult and complex knee injuries.

In a study done by Ruth Craw ford et.al ${ }^{47}$ in 2007 it was concluded that MRI is highly accurate in diagnosing meniscal and anterior cruciate ligament (ACL) tears. It is the most appropriate screening tool before therapeutic arthroscopy. It is preferable to diagnostic arthroscopy in most patients because it avoids the surgical risks of arthroscopy. The results of MRI differ for medial and lateral meniscus and ACL, with only $85 \%$ accuracy.

In a study done by TR Madhusudhan et.al ${ }^{48}$ in 2008 it was concluded that specificity, positive and negative predictive values were more favourable for clinical examination though MRI was more sensitive for meniscal injuries.

In a study done by Naranje $\mathrm{S}^{49}$ in 2008 it was concluded that in chronic ACL-deficient patients, the prevalence of posterior horn medial meniscus tears seems to be high. Anterior horn tears and radial and horizontal patterns of meniscus tears seem to be rare in chronic ACL deficiency. 
In a study done by So Yeon Lee ${ }^{50}$ in 2008 it was concluded that MRI of the knee is reliable and accurate for detection of radial tears of the medial meniscal root. coronal T2-weighted imaging is the most usefull MRI sequence.

In a study done by Vassilios S Nikolaou ${ }^{51}$ in 2008 it was concluded that MRI is very helpful in diagnosing meniscal and cruciate ligament injuries. But in a countable percentage reports with false results and in chondral defects its importance is still vague. The arthroscopy still remains the gold standard for definitive diagnosis. The accuracy for tears of the medial, lateral meniscus, anterior and posterior cruciate ligaments and articular cartilage was $81 \%, 77 \%, 86 \%, 98 \%$ and $60 \%$ respectively. The specificity was $69 \%, 88 \%, 89 \%, 98 \%$ and $73 \%$ respectively. The positive predictive value was $83 \%, 81 \%, 90 \%, 75 \%$ and $53 \%$ respectively. Finally, the clinical examination had significant lower reliability in the detection of these injuries.

In a study done by Oei $\mathrm{EH}^{52}$ in 2008 it was suggested that selective use of a short MRI examination saves costs and potentially increases effectiveness in patients with acute knee injury without a fracture on radiography.

In a study done by M. Schurz et.al ${ }^{53}$ in 2008 , it was concluded that MRI with arthroscopic findings, it is obvious that the accuracy, specificity, sensitivity, and the positive and negative predictive values are higher than in the group of the clinical examination versus arthroscopic results. These results indicate that an MRI is necessary to complete an exact clinical diagnosis in meniscus tears, but surgery should only follow an MRI, when positive clinical symptoms have been exactly identified.

In a study done by Jyrki Halinen et.al ${ }^{54}$ in 2009 , Accuracy of MRI for medial meniscal tears was $88.6 \%$, sensitivity $80 \%$, and specificity $91.2 \%$; accuracy for lateral meniscal tears was $72.7 \%$, sensitivity $55 \%$ and specificity $87.5 \%$. Accuracy and sensitivity for severity of ACL tear was $93.2 \%$ and of MCL tear $86.4 \%$. In $88.6 \%$ of the knees, bone bruises were visible, with anterolateral femoral and posterolateral tibial bone bruise being the most common. MRI revealed no chondral lesion, but arthroscopy revealed 11 chondral lesions. In combined ACL-MCL ruptures, the incidence of concomitant injuries is high and the injuries are best detected with MRI.

In a study done by Noha H. Behairy et.al ${ }^{55}$ in 2009 , study of accuracy of routine magnetic resonance imaging in meniscal and ligamentous injuries of the knee: comparison with arthroscopy done in different centres shows that MRI has high accuracy and should be used as the primary diagnostic tool for selection of candidates for arthroscopy and found a sensitivity of 47 and $100 \%$, specificity of 95 and $75 \%$ and accuracy of 73 and $78.5 \%$, respectively, for the medial and lateral meniscus. A sensitivity of $77.8 \%$, specificity of $100 \%$ and accuracy of $94 \%$ was noted for the anterior cruciate ligament (ACL). We found good kappa agreements (0.43 and 0.45) for both menisci and excellent agreement (0.84) for the ACL.

In a study done by F.Rayan et.al ${ }^{56}$ in 2009 , they compared and correlated the clinical, magnetic resonance imaging (MRI), and arthroscopic findings in cases of meniscal tear and anterior cruciate ligament (ACL) injuries and found that clinical examination had better sensitivity ( 0.86 vs. 0.76 ), specificity ( 0.73 vs. 0.52$)$, predictive values, and diagnostic accuracy in comparison to MRI scan in diagnosis for medial meniscal tears.

In a study done by Asif Rahman et.al ${ }^{57}$ in 2010, it was concluded that MRI is accurate in diagnosing meniscal injuries of knee joint and is effective in selection of patients for arthroscopy. They showed that MRI has sensitivity of $89.4 \%$ and specificity of $62 \%$ in diagnosing injuries of MM, while sensitivity of $87 \%$ and specificity of $88 \%$ in diagnosing injuries of LM. Diagnostic accuracy of MRI in MM and LM injuries was $76.4 \%$ and $88.2 \%$ respectively.

In a study done by Sharma UK et.al ${ }^{58}$ in 2011, Clinical examination showed higher sensitivity for medial meniscal tear compared to MRI, however with low specificity and diagnostic accuracy. Low field MRI showed high sensitivity, specificity, diagnostic accuracy for meniscal and cruciate ligament injury, in addition to associated derangement like articular cartilage damage, synovial thickening. The sensitivity, specificity, diagnostic accuracy of clinical examination were $96.1 \%, 33.3 \%$ and $73.1 \%$ respectively for medial meniscal tear; $38.4 \%, 96.4 \%$ and $78.1 \%$ respectively for lateral meniscal tear. The sensitivity, specificity, diagnostic accuracy of MRI were $92.3 \%, 100 \%$ and $95.1 \%$ for medial meniscal tear; $84.6 \% 96.4 \%$ and $92.6 \%$ respectively for lateral meniscal tear.

In a study done by Julian Dutka59 in 2012 , a study of arthroscopic verification of objectivity of the orthopaedic examination and MRI in intraarticular knee injury: retrospective study showed that the assessment of intra-articular knee joint lesions is a difficult diagnostic problem. In making a decision about arthroscopy of the knee joint, an appropriate sequence of examinations should be carried out, MRI and arthrosccopy. The improvement in the effectiveness of the orthopaedic examination and MRI can limit the too high frequency of diagnostic arthroscopies, which generates the risk of operation treatment and costs. The sensitivity values of 
the orthopaedic examination for injuries of the ACL, MM, LM and chondral injurieswere $86 \%, 65 \%, 38 \%$ and $51 \%$ respectively. Specificity values were $90 \%, 65 \%, 100 \%$ and $100 \%$ respectively. The MR sensitivity and specificity values were $80 \%, 88 \%, 44 \%$ and $32 \%$ and $86 \%, 64 \%, 93 \%$ and $97 \%$ respectively.

In a study done by Harish S Lokannavar60 in 2012 it was concluded that Low-field MRI alleviates the need of arthroscopy for detection of meniscus tears and ligament tears.

In a study done by S.gupta et $\mathrm{al}^{61}$ in 2012 ,showed diagnostic accuracy of MRI was $66.67 \%$ for medial meniscus and $90 \%$ for lateral meniscus GR 1 and 2 meniscal tears have low sensitivity 50\% as compared to GR 3 and 4 with $88 \%$. In the case of ACL tears, diagnostic accuracy for both clinical examination and MR examination came out to be $90 \%$ Conclusion: We can avoid diagnostic arthroscopy in patients with ACL and PCL injuries having equivocal clinical and MRI examination and go on for therapeutic modality. In case of meniscal injuries graded as 1 and 2 on MRI, are rarely seen on arthroscopy hence arthroscopy is not required for these meniscal injuries.

In a study done by Schub $\mathrm{DL}^{62}$ in 2012, study of teenagers concluded that the $3 \mathrm{~T}$ MRI studies have excellent accuracy for diagnosing ACL tears. These studies also show a higher accuracy for the diagnosis of medial meniscal tears than lateral meniscal tears. the sensitivity and the specificity of 3 T MRI were $81.0 \%$ and $90.9 \%$ for medial meniscus injuries, $68.8 \%$ and $93 \%$ for lateral meniscus injuries, and $97.9 \%$ and $98.6 \%$ for ACL injuries, respectively. The orthopedic surgeon's interpretation of $3 \mathrm{~T}$ MRI had a sensitivity and specificity of $75.7 \%$ and $92.4 \%$ for medial meniscus injuries, $69.8 \%$ and $98.3 \%$ for lateral meniscus injuries, and $100 \%$ and $98.6 \%$ for ACL injuries, respectively. Posterior horn tears had the greatest discrepancies.

In a studuy done by Kamini Gupta ${ }^{63}$ in 2013 it was concluded that MRI is a useful non-invasive modality having high diagnostic accuracy, sensitivity, and negative predictive value making it a very reliable screening test for diagnosing internal derangements. MR had $100 \%$ sensitivity and NPV of diagnosing ACL tears in this study. Clinical examination had sensitivity of $88 \%$ and NPV $75 \%$ in diagnosing ACL injuries. There was high NPV of MR examination (96\%) in diagnosing meniscus tear while the PPV of MR examination was low (71\%). These values were low in case of clinical examination.

In a study done by Subhash R Puri et.al ${ }^{64}$ in 2013 it was concluded that carefully performed clinical examination can give equal or better diagnosis of meniscal and ACL injuries in comparison with MRI scan. MRI may be used as an additional tool for diagnosis. MRI had better sensitivity ( 0.95 vs. 0.85$)$ and specificity (1.0 vs. 0.5$)$ in comparison with clinical examination for medial meniscus. In lateral meniscus injury (sensitivity 0.65 vs. 0.61 and specificity 0.95 vs. 0.92 ) and in ACL injury (Sensitivity 0.77 vs. 0.8 and specificity 1.0 vs. 0.96 ) the sensitivity and specificity of MRI versus clinical examination showed minimal difference.

In a study done by Adil Ismail Nasir et.al ${ }^{65}$ in 2013 it was concluded based on the study "The Role of Magnetic Resonance Imaging in the Knee Joint Injuries MRI is a safe, non invasive imaging modality" as MRI of the knee has been shown to be accurate in the assessment of menisci, ligaments and articular cartilage, i.e. excellent modality for assessment of soft tissue and knee joint derangements. MRI can accurately diagnose the ligament injuries of knee joint, which is an ideal technique in the diagnosis of ligament injuries of knee joint, and should be used as a routine examining method. So MRI affect the diagnosis and management of the knee injuries by decreasing the number of arthroscopic procedures, improving clinician diagnostic certainty, and assisting in management decision.MRI of the knee provides the potential for the rapid, definitive diagnosis with a non invasive examination.

In a study done by Claus Muhle et.al ${ }^{66}$ in 2013, study of diagnosis of ACL and meniscal injuries: MR imaging of knee flexion versus extension compared to arthroscopy the diagnosis of meniscal injuries, however, was not superior at both flexion positions compared to commonly performed examinations at knee extension. . The accurcy of MRI in detecting cruciate ligament tears was between $80-96 \%$ against $82-93 \%$ for clinical examination. MRI accuracy for meniscus injuries was $80-84 \%$ compared to $48-65 \%$ for clinical examination.

\section{HISTORY}

The majority of knee joint injuries results from the direct trauma to the knee joint or is caused by torsional or angulatory forces. These injuries vary in severity from simple ligamentous strain to complex injuries involving ligamentous disruption with meniscal damage and associated fracture. ${ }^{1}$ 
Since the discovery of X-rays in 1895, which marked the effective birth of diagnostic medical imaging, there have been numerous refinements of imaging techniques and the development of entire new modalities, including ultrasound, computed tomography, MRI and positron emission tomography ${ }^{8}$.

Although arthrography has the potential of a high level of accuracy, but is invasive and technically very demanding ${ }^{9}$

MRI has been proved to be reliable and safe. Since its introduction for clinical use in the mid-1980s, the MRI has emerged as a primary tool, to guide the management of knee pain. With the development of new sequences, improved signal to noise ratio, high resolution, reduced artifacts, and shorter imaging times the role of MRI in the diagnosis of patients with knee complaints has improved $^{1}$

Arthroscopy is an invasive and expensive but still considered as "the gold standard" for the diagnosis of traumatic intra-articular knee lesions, having accuracy as high as $95 \%$ to $98 \%$.and with a single intervention both diagnosis and repair of ligamentous and meniscal injuries can be done.

\section{NORMAL ANATOMY OF KNEE JOINT}

The knee is the largest joint in the body. It is a complex 'hinge' joint made up of the lower end of the femur, the upper end of the tibia and the patella, which slides in a groove on the end of the femur. The knee joint has three components, the lateral tibiofemoral, medial tibiofemoral and patellofemoral joints. Four bands of tissue, the anterior and posterior cruciate ligaments, and the medial and lateral collateral ligaments connect the femur and the tibia and provide joint stability.

Strong thigh muscles give the knee its strength and mobility. The surfaces where the femur, tibia and patella come in contact are covered with articular cartilage, a smooth substance that cushions the bones and enables them to glide freely. Semicircular rings of tough fibrous-cartilage tissue called the lateral and medial menisci act as shock absorbers and the bones of the knee are surrounded by a thin, smooth tissue capsule lined by a thin synovial membrane which releases a special fluid that lubricates the knee, reducing friction to nearly zero in a healthy knee (Fig-1).

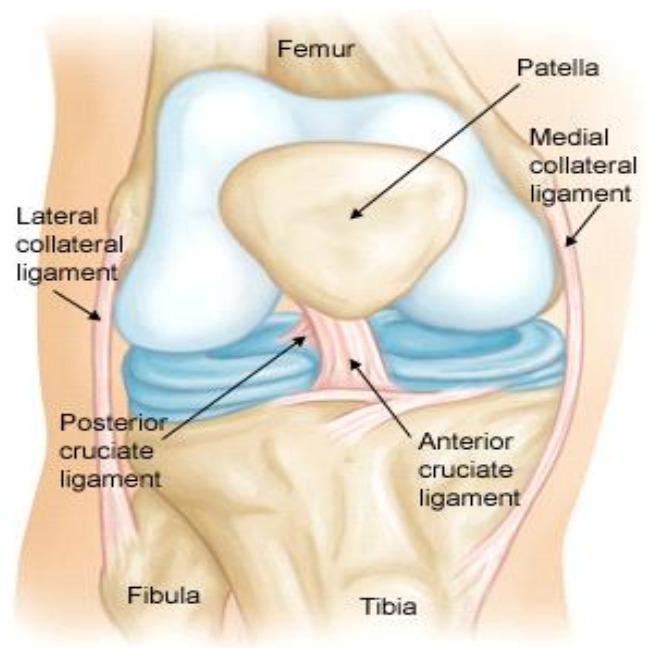

Fig-1 : Normal knee joint

\section{TYPE OF JOINT:}

It is a synovial modified hinge joint, which also permits a small degree of rotation. In the knee joint, the femoral and tibial condyles articulate, as does the patella and patellar surface of the femur.

Fibula does not contribute to the knee joint.

Fibrous capsule: 
It is attached superiorly to the femur about $1 \mathrm{~cm}$ above the articular margin with three special features. It is attached to the margins of the articular surfaces except anteriorly, where it dips downwards. In the anterior part of the capsule there is a large opening through which the synovial membrane is continuous with the suprapatellar bursa. This bursa extends superiorly three finger breadths above the patella between the femur and quadriceps. Posteriorly, the capsule communicates with another bursa under the medial head of gastrocnemius and often, through it, with the bursa of semimembranosus. Posterolaterally, another opening in the capsule permits the passage of the tendon of popliteus. The lateral expansions extend backwards on each side and downwards to the tibia forming medial and lateral patellar retinacula. On its deep surface, it is attached to periphery of each meniscus and connects it to adjacent margin of head of tibia; this connection is termed as coronary ligament.

\section{Ligamentum patellae:}

It is strong, flat, ligamentous band, about $8 \mathrm{~cm}$ in length. It is the central portion of common tendon of the quadriceps femoris, which is continued from the patella to the tuberosity of the tibia. The ligamentum patellae is related to the superficial and deep infrapatellar bursa and also to the infrapatellar pad of fat.

\section{Extra capsular ligaments:}

The capsule of knee joint is reinforced by extra capsular ligaments

The oblique popliteal ligament: This is an expansion from the tendon of semimembranosus.(Fig-2)

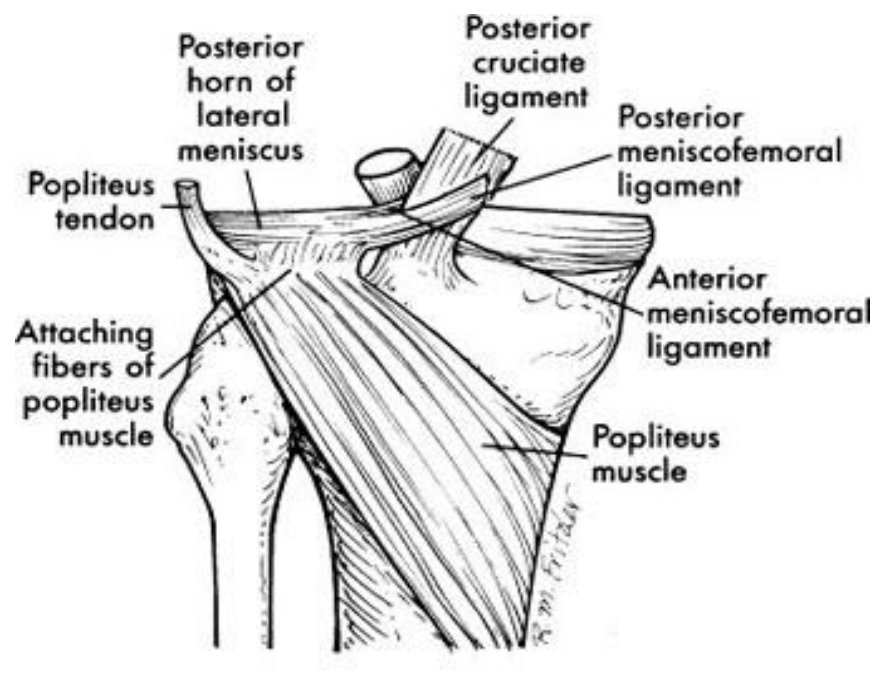

Fig-2 Extracapsular ligaments of knee joint

It runs upwards and laterally blending with the posterior surface of the fibrous capsule. It gets attached to the intercondylar line and the lateral condyle of femur.

The arcuate popliteal ligament: It has Y shaped fibers, the stem of which is attached to the head of fibula. The posterior limb arches medially over tendon of popliteus to be attached to the posterior border of intercondylar area of tibia. The anterior limb, which is sometimes absent, extends to lateral epicondyle of femur where it is connected with lateral head of gastrocnemius. It is often termed short lateral ligament.

The fibular collateral ligament: It is a strong, rounded cord, attached above, to the lateral epicondyle of the femur, immediately above the groove for the tendon of the popliteus and below, to the head of the fibula, in front of the apex. The greater part of it is hidden by the tendon of biceps femoris, which embraces and is attached to the ligament. The ligament has no attachment to the lateral meniscus.

The tibial collateral ligament: It is a broad, flat band, situated nearer to the back than to the front of the joint. It is attached above, to the medial epicondyle of the femur immediately below the adductor tubercle; below, to the medial condyle and medial surface of the shaft of the tibia. It consists of anterior and posterior parts. The anterior part is flattened band and about $10 \mathrm{~cm}$ long and is easily distinguished from the fibrous capsule which lies deep to it; one or more bursae may separate it from the fibrous capsule 
and medial meniscus. It is attached below to the medial border and posterior surface of the medial surface of tibia. It covers the medial geniculate vessels and nerve and the anterior part of the tendon of semimembranosus. It is crossed below by the tendons of sartorius, gracilis and semitendinosus. The posterior part of the ligament is short and blends with the capsule and the medial meniscus. It is attached to the medial surface of the tibia above the groove for the semimembranosus.

Intra capsular ligaments/cartilages: The cruciate ligaments and menisci are enclosed within the knee joint. (Fig-3)

The anterior cruciate ligament: It passes from the front of the intercondylar area of the tibia to the medial side of the lateral femoral condyle. This ligament prevents hyperextension and resists forward movement of the tibia on the femur.

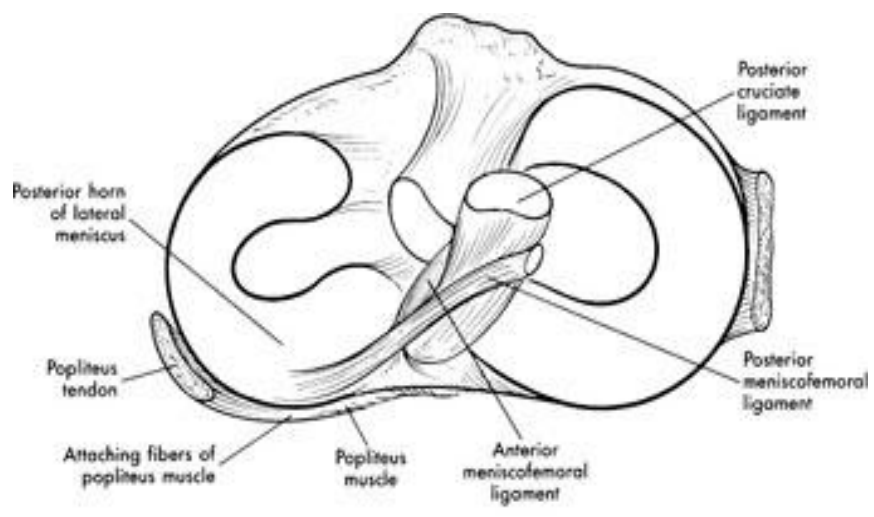

Fig-3 Intracapsular ligaments of knee joint

The posterior cruciate ligament: It passes from the back of the intercondylar area of the tibia to the lateral side of the medial condyle. It becomes taut in hyperflexion and resists posterior displacement of the tibia on the femur.

The menisci (semi-lunar cartilages): These are crescentic fibro-cartilaginous 'shock absorbers' within the joint. They lie within deepened grooves on the articular surfaces of the tibial condyle. The medial meniscus is $\mathrm{C}$ shaped and larger than the lateral meniscus. It is broader and thicker posteriorly. The lateral meniscus is smaller, thicker and forms a complete ring. Either meniscus may occasionally appear discoid with thickened periphery extending into normally thinning centre. The menisci are attached to the tibial intercondylar area by their horns and around their periphery by small coronary ligaments. The lateral meniscus is loosely attached to the tibia and connected to the femur by two meniscofemoral ligaments.If dominant part is anterior, it is called anterior meniscofemoral ligament of Humphrey (anterior to the PCL) and if posterior part is dominant it is known as meniscofemoral ligament of Wrisberg (posterior to the PCL). The outer margin of medial meniscus is blended with fibrous capsule and deep surface of the medial collateral ligament. The lateral surface of the lateral meniscus is grooved by, and blends with, the tendon of popliteus, which is separate from fibular collateral ligament. The transverse ligament joins the anterior ends of menisci. Functions of menisci include the distribution of stresses over the articular cartilage, the absorption of shocks during axial loading, the stabilization of the joint in both flexion and extension, and joint lubrication; they also make a minor contribution toward secondary stabilization of the knee after cruciate ligament injuries. Because of their nerve supply, they give rise to propioceptive impulses.

The transverse ligament: It connects the anterior convex margin of the lateral to the anterior end of medial meniscus; its thickness varies in different subjects, and is sometimes absent.

Synovial Membrane: The synovium is a thin membrane that lines the knee capsule and attaches to the margins of the articular surfaces and the periphery of the fibrocartilaginous menisci (Fig-4).

Small gap exists between the insertion of the synovial membrane/capsule and the nearby articular cartilage, effectively resulting in a "bare area." Anteriorly, the synovial lining extends superiorly, above the patella and deep to the quadriceps femoris, to form the suprapatellar bursa, held in position by a small muscle, the articularis genus, arising from the vastus intermedius. The synovial membrane envelops the anterior and posterior cruciate ligaments so as to exclude them from the synovial cavity (intra-articular but extrasynovial). Posteriorly, the synovial membrane extends caudally on the deep surface of the popliteus tendon, forming the popliteal bursa. An additional bursa, the semimembranosus bursa, lies between the medial head of the gastrocnemius and medial femoral condyle, and also communicates frequently with the synovial cavity of the joint. 


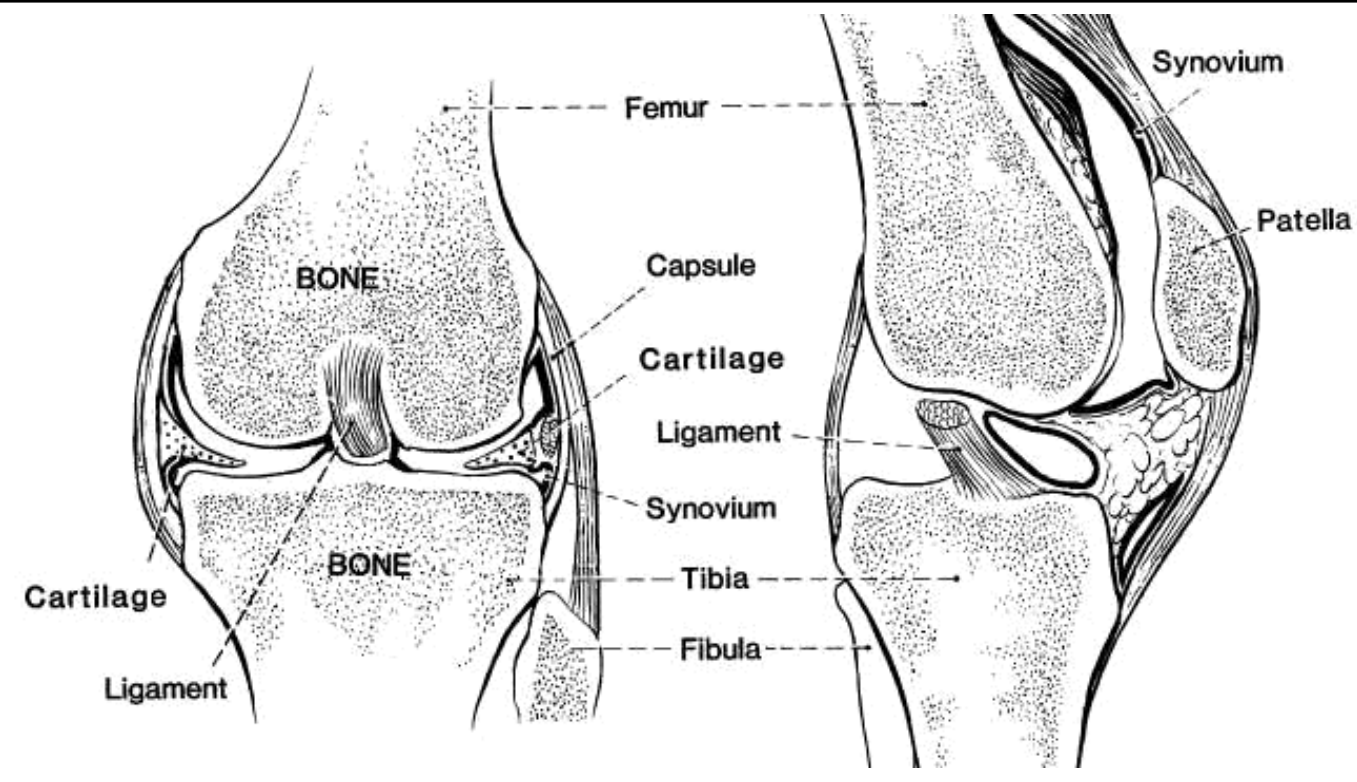

Fig-4 Synovial membrane and fossa of knee joint

Bursae around the Knee Joint: As many as thirteen bursae have been described around the knee joint- four anterior, four lateral and five medial. These are as under;

\section{Anterior}

- Subcutaneous prepatellar bursa

- Subcutaneous infrapatellar bursa

- Deep infrapatellar bursa

- Suprapatellar bursa

\section{Lateral}

- A bursa deep to the lateral head of gastrocnemius.

- A bursa between the fibular collateral ligament and the biceps femoris.

- A bursa between the fibular collateral ligament and the tendon of popliteus.

- A bursa between the tendon of popliteus and the lateral condyle of tibia.

\section{Medial}

- A bursa deep to the medial head of gastrocnemius

- The anserine bursa is a complicated bursa which separates the tendons of sartorius, gracilis and semitendinosus from one another, the tibia and from the tibial collateral ligament.

- A bursa deep to the tibial collateral ligament

- A bursa deep to the semimembranosus

- Occasionally a bursa is present between the semimembranosus and the semitendinosus.

\section{RELATIONS OF KNEE JOINT:}

- Anteriorly: Quadriceps femoris, patellar retinacula and supra patellar retinacula and suprapatellar bursa.

- Posteriorly: Oblique popliteal ligament, popliteus, popliteal artery with vein posterior and tibial nerve posterior to both; lymph nodes; heads of gastrocnemius, lower end of semimembranosus and semitendinosus.

- Posteromedially: Sartorius and gracilis tendons.

- Posterolaterally: Biceps femoris with the common peroneal nerve on its medial side.

\section{MOVEMENTS OF KNEE JOINT:}

Flexion and extension are the principal movements at the knee. Some rotation is possible when the knee is flexed but is lost in extension. During the terminal stages of extension the large medial tibial condyle screws forwards onto the femoral condyle to 
lock the joint. Conversely, the first stage of flexion is unlocking the joint, by internal rotation of the medial tibial condyle, an action performed by popliteus.

The principal muscles acting on the knee are,

- Extension: Quadriceps femoris.

- Flexion: Predominantly the hamstrings but also gracilis, gastrocnemius and sartorius.

- Medial Rotation: Popliteus, semitendinosus and semimembranosus bring about medial rotation of the flexed leg assisted by the sartorius and gracilis.

- Lateral Rotation: Lateral rotation of the flexed leg is brought about by the biceps femoris.

During the last 30 degrees of extension, medial rotation of the femur occurs so that the articular surfaces of femur and tibia are completely used up. This is called locking of the knee. Similarly during initial stages of flexion, lateral rotation takes place, which unlocks the knee joint. This is brought about by the action of popliteus. Locking of knee helps the knee to remain in the position of full extension without much muscular effort.

\section{DIAGNOSIS OF KNEE TRAUMA}

Internal derangements of traumatic knee can be diagnosed by following imaging techniques

- Ultrasound

- Arthrography

- Computed Tomography

- Arthroscopy

- Magnetic Resonance Imaging

\section{ULTRASOUND}

Ultrasound examination has been widely used for detecting musculoskeletal disorders in recent decades. Sonographic examination can accurately detect effusion of the knee. The detection of knee effusion in patients with traumatic knee injury by ultrasound examination is highly indicative of internal knee derangement. It has the advantages of being noninvasive, readily available, dynamic, and allows for good visualization of superficial structures. It is well accepted for use in evaluating extraarticular structures of the knee, but is still debated in intra-articular examination.

However, the clinical role of sonographical examination in detection of knee effusion is not well explored.

\section{ARTHROGRAPHY}

It can be used to improve the accuracy of diagnosis.It is invasive and can cause complications. It requires a skilled person for interpreting and reporting the results. It also involves risk of exposure to radiation.

\section{COMPUTED TOMOGRAPHY}

CT provides valuable information about co-existing joint effusion, chondral and osseous fractures and can accurately locate intra articular loose bodies. It also provides a non-invasive method of assessing the cruciate ligaments by thin section CT with reformatting in sagittal plane. This method however is not precise and exposes the patient to ionizing radiations.

\section{ARTHROSCOPY}

Arthroscopy is considered as "the gold standard" for the diagnosis of traumatic intraarticular knee lesions, having accuracy as high as $95 \%$ to $98 \%$. However, arthroscopy is an invasive and expensive tool that requires hospitalization and regional or general anesthesia, thus presenting all the potential complications of an open surgical procedure. Today, arthroscopy of knee joint is the most common procedure performed among orthopedic surgeons. Initially, knee arthroscopy was used as a diagnostic technique. With the advent of magnetic resonance imaging and other non-invasive techniques, knee arthroscopy is now used for the 
treatment of various knee pathologies. Indications for knee arthroscopy include the treatment of meniscal pathology, specified articular cartilage lesions, osteochondral lesions, loose bodies, advanced synovitis, cruciate ligament tears and certain tibial plateau fractures. One of the most common indications for knee arthroscopy has historically been the debridement of the arthritic knee.

\section{MAGNETIC RESONANCE IMAGING}

MRI has been confirmed as the ideal approach for primary diagnosis of traumatic intra-articular knee lesions. It is noninvasive, fast, can be done on an outpatient basis, and is free of complications. It is unique in its ability to demonstrate the nature and extent of injuries. Imaging can be accomplished in any plane without moving the patient and a wide variety of MRI pulse sequences can be performed to produce diagnostic quality images. These include spin echo, fast (turbo) spin-echo, and gradient-echo sequences, which all have been proven suitable for knee imaging. T1 or proton density-weighted sequences are most suitable for visualizing the ligamentous anatomy.

Typically, a routine scanning protocol would consist of a combination of one or more of these sequence types performed in the axial, sagittal, and coronal planes using thin sections (maximum $3 \mathrm{~mm}$ with an interslice gap of 0.5 to $1 \mathrm{~mm}$ ).

\section{MR ANATOMY OF KNEE JOINT}

MRI of knee is performed using transmit / receive general purpose extremity surface coil. Quadrature and phase array coils are also available. Increased spatial resolution and decreased signal to noise ratio are significant advantages of these coils.

Positioning: Imaging is done with full extension in neutral position. A 14 to $16 \mathrm{~cm}$ field of view and a 3-4mm slice thickness. MRI is obtained in the Axial, Sagittal and coronal views.

Pulse Sequences: A wide variety of MRI pulse sequences can be performed to produce diagnostic quality images. These include spin echo, fast (turbo) spin-echo, and gradient-echo sequences, which all have been proven suitable for knee imaging. T1 or proton density-weighted sequences are most suitable for visualizing the ligamentous anatomy. T2 or STIR sequences with fat saturation are essential to demonstrate bone marrow edema. Typically, a routine scanning protocol would consist of a combination of one or more of these sequence types performed in the axial, sagittal, and coronal planes using thin sections (maximum 3 mm with an interslice gap of 0.5 to $1 \mathrm{~mm}$ ). A field-of-view of 12 to $16 \mathrm{~cm}$ depending on patient size is commonly used with a highresolution matrix of at least 140 steps in the phase-encoding direction and 256 steps in the frequency-encoding direction.

Synovial Membrane: It appears on both sagittal and coronal images as linear medium signal intensity on T1 weighted and proton density images and as high signal intensity on T2 weighted images.

Bursae around the knee joint: They are not usually seen on MRI unless they are inflamed and fluid filled.

Anterior Cruciate ligament: ACL is best seen on sagittal, oblique images with slices oriented parallel to the cortex of the lateral femoral condyle (Fig 5). It may appear as a solid band or as three or four separate low signal intensity bands. The tibial attachment is usually better seen than the femoral attachment because of partial volume averaging with lateral femoral condyle. Signal intensity on T1 weighted and spin density images at the tibial insertion is increased. The anterior border of taut ACL should nearly parallel the roof of the intercondylar notch. Coronal and axial images are useful in confirming the findings made on sagittal images. Coronal images show the ACL as a curvilinear fan like structure adjacent to the horizontal segment of PCL, near the medial surface of the lateral femoral condyle. Axial images depict ACL as low signal band that is flattened against the medial surface the lateral femoral condyle. All imaging sequences demonstrate fat at the intercondylar notch. 


\section{JMSCR Vol||3||Issue||12||Page 8590-8631||December}

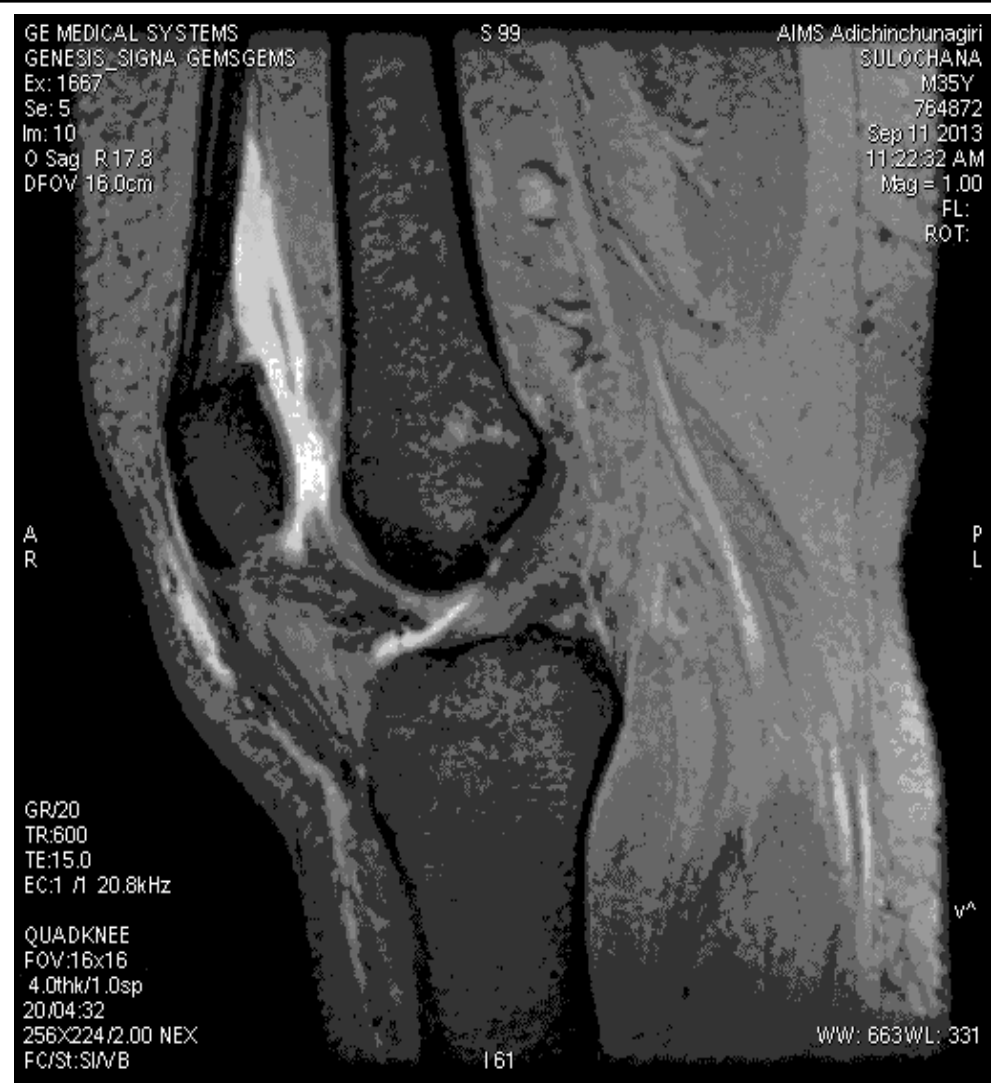

Fig- 5 Sagittal gradient image of the knee - Intact ACL .

Posterior Cruciate Ligament: Sagittal images best depict the PCL, which appears as a uniformly low signal intensity structure with a nearly horizontal take off at the femoral origin and then and abrupt descent at about 45 degrees to the tibia (Fig-6). This angled portion of the ligament is normally directed towards the femur. The meniscofemoral ligaments of Humphrey and Wrisburg are seen as low signal intensity dots anterior and posterior to the PCL and should not be mistaken for displaced meniscal fragments or an intact PCL in presence of tear.

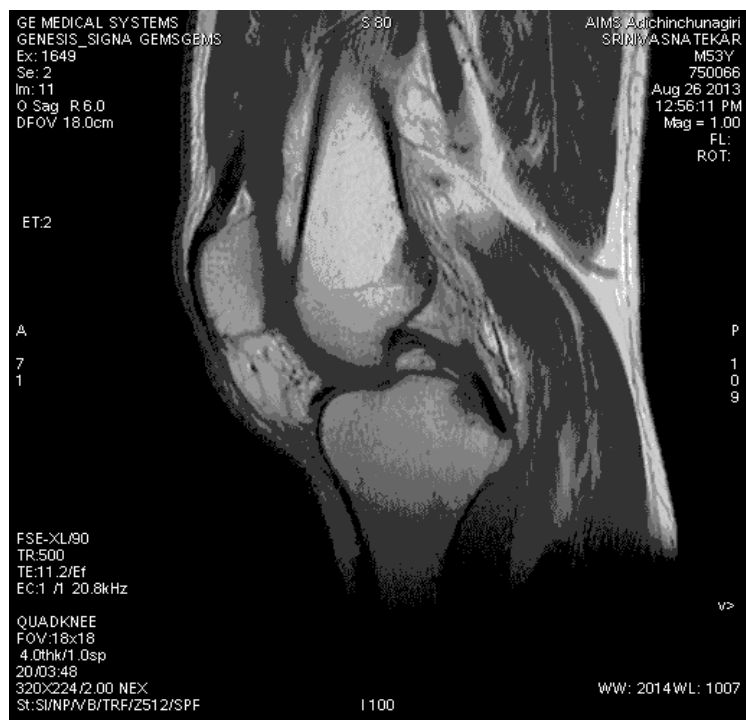

Fig -6 Normal PCL- T2 WI sagittal view of the knee - homogeneous low-signal PCL.

Medial and Lateral Collateral Ligament: It is best seen on coronal images where it appears as homogenously low signal intensity structure on all pulse sequences. Moderately increased signal intensities may be seen between and superficial and deep fibers and below the superficial fibers at the distal tibial attachment site, where fat is normally interposed (Fig-7). 


\section{JMSCR Vol||3||Issue||12||Page 8590-8631||December}

Oblique Popliteal Ligament (Posterior Oblique Ligament): It is best seen on coronal and axial images. A coronal oblique plane along the superoinferior course of the OPL (POL) is also optimal for imaging.

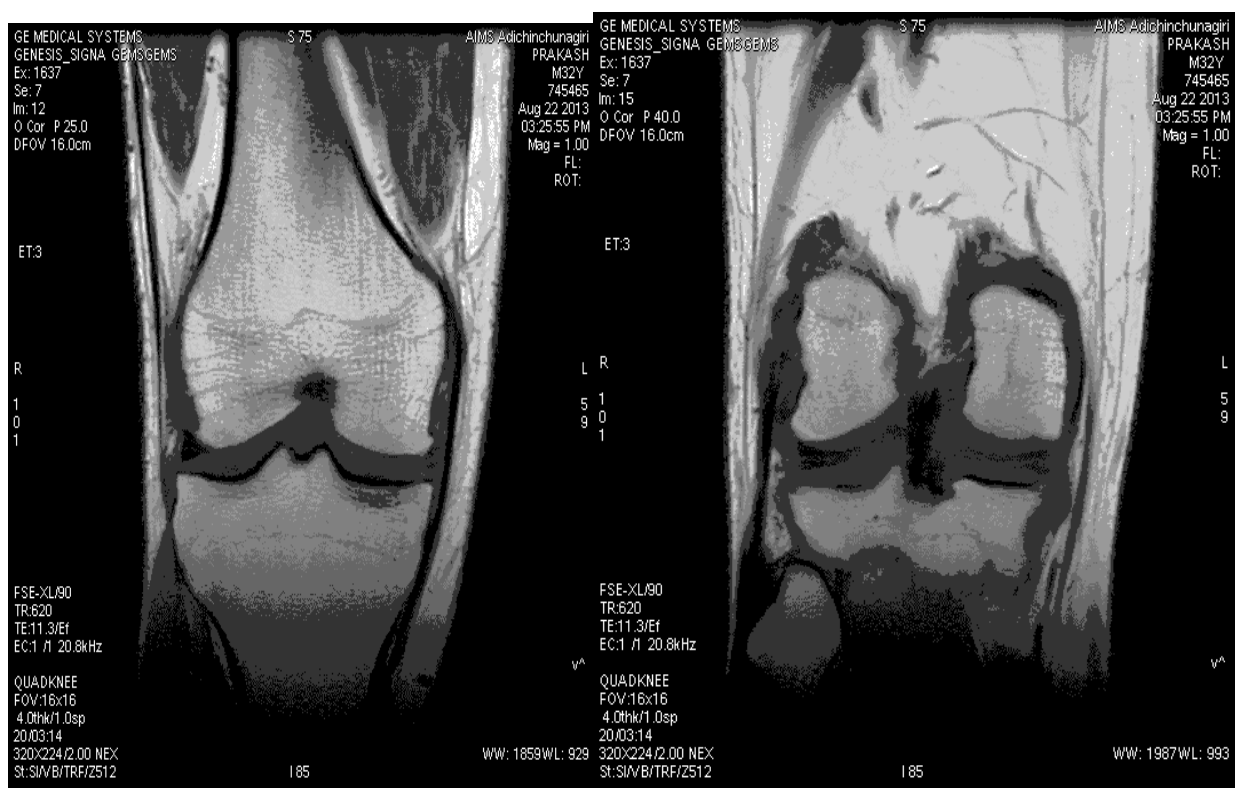

A Fig-7 B

Fig A. Medial collateral ligament and distal end of fibular collateral ligament. B. Proximal fibular collateral ligament in Coronal T1 WI is well demonstrated.

The Menisci: The sagittal plane is the most important plane in assessing the menisci, with a coronal plane providing supportive rather than new information and the axial plane increasing the accuracy of the sagittal and coronal planes when combined. In general, sagittal images optimally show anterior and posterior horns of the medial and the lateral menisci, coronal images help in evaluating the Meniscal bodies and thin axial sections provide an additional view of menisci and their free edges.

Sagittal image: The anterior and the posterior horns of menisci appear as isosceles triangles. The posterior horn of medial meniscus is twice the size of anterior horn. The anterior and posterior horns of lateral meniscus are of same size. The posterior horn of either menisci should never appear smaller than the anterior horn. On both sides, the menisci appear as flat bands. On lateral side, the more central the slices take on bowtie configuration because of smaller radius of curvature. (Fig-8)
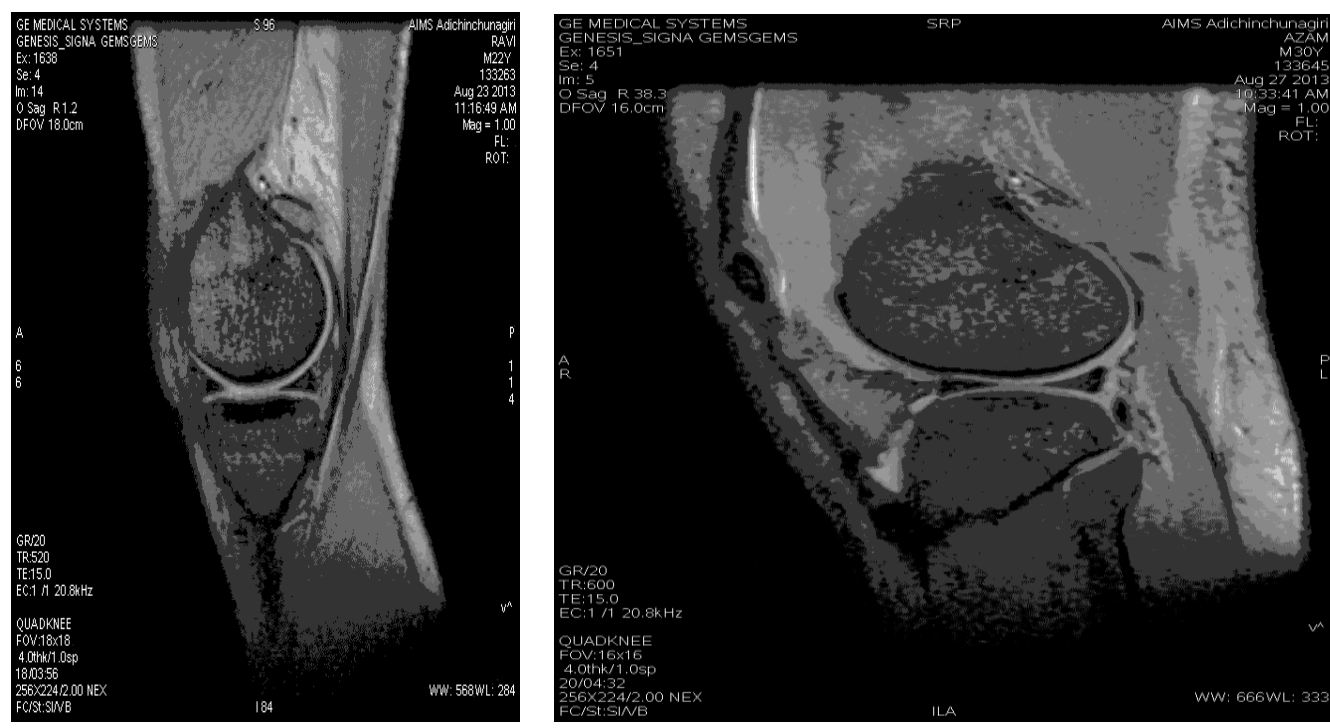

A Fig-8 B

(A) Sagittal GRE image of a normal medial meniscus. (B) Sagittal GRE image of a normal lateral meniscus. 
Coronal images: Mid portion of the knee produce best images of bodies of both menisci. They appear triangular and slightly larger laterally than medially. The capsular attachment on the medial side is incorporated in the tibial and medial collateral ligament. A small amount of fat may be interposed between the body of the medial meniscus and the capsule. On posterior coronal cross sections, the posterior horns appear as flat bands. On lateral cross sections, the popliteal tendon courses upward and laterally at 45 degrees. More anteriorly, the anterior horn of lateral meniscus appears as a band like structure. The anterior horn of medial meniscus extends more anteriorly than that of lateral meniscus.

The medial and lateral menisci, the transverse ligament, and the meniscofemoral ligament appear homogenously dark on all pulse sequences. The vascular and avascular zones cannot be distinguished on MRI ${ }^{[31]}$. The vascularized zone does not demonstrate enhancement with intravenous gadolinium.

\section{PATHOGENESIS AND MAGNETIC RESONANCE IMAGING APPEARANCES OF INTERNAL DERANGEMENTS OF KNEE}

The traumatic internal derangements of the knee can be classified as those involving,

- Cruciate ligaments

- Collateral ligaments

- Menisci

- Extensor mechanism and the patellar tendon

- Osseous and osteochondral injuries

\section{THE CRUCIATE LIGAMENTS}

\section{Anterior Cruciate Ligament}

Most common mechanism of injury to anterior cruciate ligament is external rotation of femur on a fixed tibia with a valgus force. Other less common mechanisms include hyperextension, internal rotation with maximal extension or anterior translation of tibia produced by a direct force against the back of calf. Clinically the patients present with hemarthrosis and an audible pop at the time of injury. The anterior cruciate ligament has limited healing capacity possibly related to heterogeneity of collagen fibers or fibroblast function within a synovial environment.

Acute rupture of the ACL ranges from low grade, partial thickness to full thickness and are located most commonly in the mid- to proximal aspect of the ligament. ACL tears occur up to eight times more commonly in females than in males. A torn ACL fiber has increased T2-weighted signal and an abnormal contour. In some full-thickness tears, an amorphous mass replaces the discrete ACL fibers. Fluid can fill the gap between the fibers of a full-thickness tear. The location of the tear can be described as proximal, midsubstance, distal, or involving the femoral or tibial attachment.Women are more likely to have tears involving the proximal ACL, likely because of the higher incidence of non-contact inciting mechanisms. Avulsion of the ACL from the tibial attachment site is more common in young patients.

On MR imaging there are both direct and indirect signs of anterior cruciate ligament injury.

The direct signs include

- Discontinuity of the fibers of anterior cruciate ligament

- An abnormal contour of anterior cruciate ligament.

The indirect signs include ${ }^{[25,33]}$

- Angulation of the posterior cruciate ligament of less than 105o,

- Anterior tibial translation exceeding $6 \mathrm{~mm}$

- Overhanging posterior horn of the lateral meniscus by $2.5 \mathrm{~mm}$

- Deep lateral femoral notch exceeding $2 \mathrm{~mm}$ in depth 
- Segond fracture of the lateral tibia involving the middle portion of the lateral fibrous capsule at the meniscofemoral attachment

- Chip fracture of the posterior tibia

- Rotary bone contusion pattern.

- Irregularity of the free concave edge of Hoffa's fat pad suggestive of synovitis

The site of an ACL tear is easier to identify during the sub acute phase. The "double PCL" sign classically is seen during this phase with the distal end of the torn ACL anterior PCL. Occasionally, the proximal end of the ACL may be displaced posterior to the PCL, simulating a large, loose body.

Chronic ACL tears are seen in several different ways on MRI. If the ligament has completely atrophied, it will not be visualized. There may be a small attachment on the tibia left in chronic tears. The distal end of the ACL may also be intact and attached to the PCL without visualization of the proximal end. This is commonly mistaken for an intact ligament on MRI.

Posterior Cruciate Ligament

The PCL is injured by one of three mechanisms:

- Knee hyperextension,

- Knee hyper flexion, or

- Posterior displacement of the tibia with the knee flexed.

The PCL most commonly tears at the mid portion of the ligament, and is more likely than the ACL to have a partial tear. PCL injuries have a high association with other injuries. Trabecular microfractures, or bone bruises are seen associated commonly. PCL tears are also associated with posterolateral corner injuries.

Acute tears of the PCL are usually manifested by thickening of the middle portion of the ligament with increased signal on both T1- and T2- weighted images. The mass like quality that an acutely torn ACL exhibits is not seen in acute PCL tears. Also, unlike acute ACL tears, continuity of the ligament is maintained in the PCL with acute trauma.

Chronic tears of the PCL are difficult to diagnose and image. Because most PCL injuries heal without surgery, the ligament will form fibrous scar in the place of normal ligament. This scar generally has the same signal intensity of native tissue. Secondary signs such as a lax ligament or persistent increased signal may help diagnose chronic PCL tears.

\section{THE COLLATERAL LIGAMENTS}

\section{Medial Collateral Ligament (MCL)}

MCL injuries are usually caused by valgus stress, and are often associated with other knee derangements. ${ }^{[38]}$ The combination of an ACL tear, MCL tear, and medial meniscal tear occurs with valgus stress to the knee while the foot is fixed on the ground, and is also known as the “unhappy triad” or O’Donoghue's triad.

The traditional classification of MCL tears is to grade the damage as first- through third-degree sprain/tear injuries.

- Grade I: Lesions are defined as high signal intensity superficial to the MCL representing edema, with intact MCL fibers.

- Grade II: Lesions in which fluid signal extend partially through the MCL, although some fibers remain intact

- Grade III: Lesions with complete discontinuity of the MCL fibers seen along with surrounding edema, consistent with a complete rupture.

\section{Lateral Collateral Ligament (LCL)}

Almost all tears of the LCL are associated with other injuries. These injuries include damage to structures within the region of the LCL, such as the capsule, the biceps femoris, and the popliteus, as well as to structures elsewhere in the knee, such as the cruciate 
ligaments or the lateral tibial rim (Segond fracture) Like the MCL, the LCL is best visualized on coronal images. Lateral collateral ligament injuries are usually classified similarly as medial collateral ligament lesions, using the 3-point grading system as outlined above. Lesions of the LCL are most often caused by varus stress and therefore may be associated with impression fractures of the medial tibial plateau. In addition, LCL ruptures are frequently seen together with other injuries of the posterolateral corner.

\section{iii) THE MENISCI}

Abnormal shearing forces may be generated during compression and rotation of knee resulting in meniscal damage. The rotation of femur on a fixed tibia during flexion and extension places the menisci at risk for injury. Clinical signs of meniscal injury include joint pain along the joint line, giving way, clicking, locking of knee in fixed flexion and effusions. Before embarking upon the pathological states, it is worthwhile considering normal anatomic variants which should not be mistaken for pathological conditions.

Before embarking upon the pathological states, it is worthwhile considering normal anatomic variants, which should not be mistaken for pathological conditions.

\section{NORMAL MENISCAL VARIANTS:}

The Discoid Meniscus: It is a congenital anomaly found usually in lateral meniscus. It has a reported incidence of 0.4 to $16.6 \%$. A meniscus is said to be discoid if there is continuity between anterior and posterior side [bow tie appearance] on five or more contiguous $3 \mathrm{~mm}$ sagittal MRI section. It presents with symptoms of joint line tenderness, "snapping", and locking of the knee. The three types of discoid lateral meniscus are complete, incomplete, and the Wrisberg variant. Some investigators include a ringshaped meniscus's a fourth type. The complete and incomplete types have a firm, normal posterior tibial attachment and are stable. Symptomatic patients who have these types of discoid menisci usually are treated with a partial meniscectomy. In contrast, the Wrisberg variant has no posterior coronary or capsular attachments and increased T2 signal is present between the meniscus and the capsule, simulating a peripheral tear or a fascicular injury. The Wrisberg variant has the most notable symptoms, with a "'snapping' sensation occurring when the posterior horn moves across the femoral condyle during flexion and extension.

A discoid medial meniscus is much less common, with the incidence reported to be $0.12 \%$ to $0.6 \%$.

On MR, the diagnosis of a discoid meniscus is suggested by identifying either meniscal tissue on three continuous sagittal 5-mmthick slices, or a meniscal body on coronal images greater than $15 \mathrm{~mm}$ wide or extending into the intercondylar notch. The discoid meniscus has an increased incidence of tears and degeneration, likely caused by its abnormal shape, resulting in increased stress on the meniscus. Intrasubstance" grade 2" signal, or abnormal signal not extending to an articular surface, is noted in $24 \%$ of discoid menisci and is more common in complete discoid menisci. Typically, this abnormal signal is not considered clinically significant. However, in the population with discoid menisci, some investigators report that this Intrasubstance signal may besignificant clinically.

Meniscal Ossicles: They are reported in $0.15 \%$ of patients and are thought to be either developmental or posttraumatic. These small, ossific foci are found typically in the posterior horn of the medial meniscus and are associated with meniscal tears. They can be asymptomatic, or associated with pain and a sensation of locking, clinically simulating a torn meniscus with a flap component. The ossicle follows the signal of bone marrow on MRI.

Meniscal flounce: It is a wavy appearance along the free edge of the meniscus. Previously, meniscal flounce was thought to be identified only at arthroscopy, in the presence of joint fluid in the setting of an ACL or medial collateral ligament (MCL) tear. However, a flounce can be seen without a ligament injury. Recently, the Meniscal flounce has been identified with MR imaging when the knee is in 10 degrees of flexion.. The flounce can appear truncated on coronal images and can simulate a tear or degeneration. The incidence at MR is reported to be from $0.2 \%$ to $6 \%$. A flounce-like appearance can be seen with meniscal tears.

Meniscal Extrusion: It is measured from the outer meniscal edge to the proximal tibial margin.Extrusion of the medial meniscus more than $3 \mathrm{~mm}$ is considered abnormal. This degree of extrusioncan be seen in patients who have advanced Meniscal degeneration, and various types of meniscal tears. Although extrusion of the anterior horn or body of the lateral meniscus sometimes is considered a normal variant, others consider extrusion of the lateral meniscus more than $1 \mathrm{~mm}$ to be abnormal. 
MENISCAL TEARS

The cause of meniscal tears can be divided into two categories.

- Increased force on a normal meniscus, usually resulting in longitudinal or radial tears, and

- Normal forces on a degenerative meniscus, usually producing horizontal tears in the posterior half of the meniscus.

Tears are more common in the medial meniscus, possibly because the medial meniscus is less mobile, and it bears more force during weight bearing than the lateral meniscus, with $56 \%$ of tears involving the posterior horn of the medial meniscus. Tears isolated to the anterior two thirds of the meniscus are uncommon, representing only $2 \%$ of medial and $16 \%$ of lateral meniscal tears.

Lateral meniscal tears are more common in younger patients (under 30 years old), who have a higher incidence of tears related to sporting events than do older patients. It is likely that this is related to the higher incidence of concomitant ACL tears in this population.

\section{Diagnostic Criteria:}

Two criteria for diagnosing a meniscal tear are commonly used:

- An intrasubstance area of intermediate or high signal intensity that unequivocally extends to the articular surface,

- Abnormal meniscal morphology

i) Intrasubstance signal: It is graded as follows:

Grade 1: Intrameniscal high signal intensity of irregular or globular appearance that is confined within the meniscus and does not extend to the articular surface.

Grade 2: The signal is linear and does not intersect the inferior or superior articular surface. It may, however, contact the capsular margin at the posterior aspect of the meniscus.

Grade 3: Tears characterized by linear high or intermediate signal intensity that extends to the superior and/or inferior articular surface.

Grade 4: It is sometimes added to indicate a complex tear with multiple components or fragmentation.

Both grade 1 and grade 2 lesions do not represent a tear, but indicate mucinous and mucoid intrasubstance degenerative change and are usually encountered after the third or fourth decade. In children and adolescents, prominent vasularity may resemble grade 1 or grade 2 lesions.

ii) Abnormal Meniscal morphology:

Morphologic changes of the meniscus associated with meniscal tears include blunting of tip of the inner free meniscal edges of the meniscus, displacement of a portion of the meniscus, interrupted appearance of the meniscus and abnormal size of a segment of the meniscus is detached (a bucket handle tear), the remaining peripheral portion appears small and often truncated. The displaced portion usually lies within the intercondylar notch beneath the PCL. Coronal views are helpful in further identifying such displaced fragments. An abrupt change in contour of the meniscus, known as the 'notch' sign, is an important indicator of a meniscal tear. Although the normal meniscal flounce can simulate it, the presence of abnormal intrameniscal signal makes the notch sign a more definite indicator of a meniscal tear.

Classification of tears:

1. Horizontal tears

2. Vertical tears

3. Complex tears 
1. Horizontal Tears: Horizontal tears are parallel to the tibial plateau and divide the meniscus into upper and lower segments. The Cleavage type extends to the apex and divides the menisci into upper and lower halves. They are referred to as 'fish mouth' tears. In the Non cleavage type, tear extends to the superior and inferior articular surface of the meniscus. Horizontal tears are most common within the posterior horn of the medial meniscus. Linear horizontally oriented grade II signal intensity is sometimes mistaken for complete horizontal tears (Fig-9).

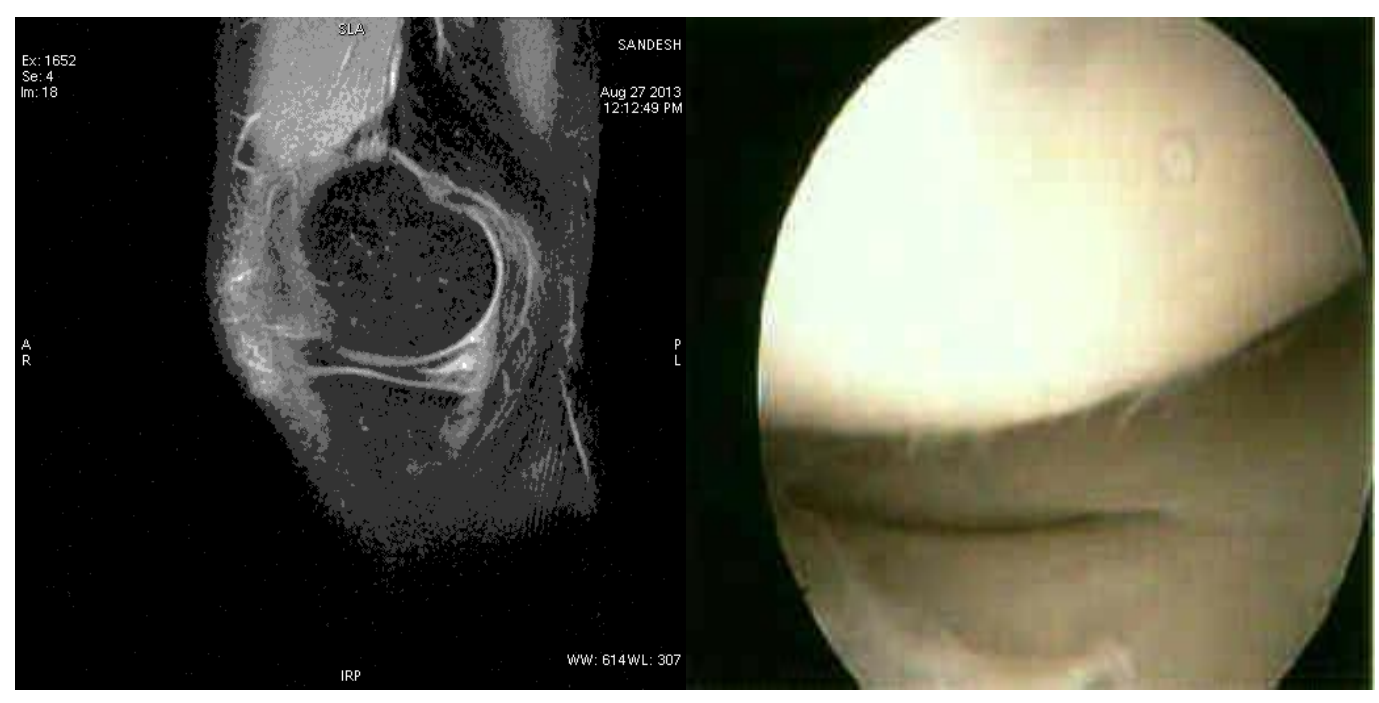

A Fig-9 B

(A)Sagittal PD FS and (B)arthrosccopy image demonstrating a horizontal tear of the posterior horn of the medial meniscus.

2. Vertical Tears: They are perpendicular to the tibial plateau.

A vertical longitudinal tear occurs between the circumferential collagen fibers parallel to the long axis of the meniscus. Longitudinal tears can be obliquely oriented, but are not parallel to the tibial plateau. Vertical grade III signal intensity located within the peripheral third of the meniscus is considered a peripheral longitudinal tear.

A vertical radial tear occurs perpendicular to the circumferential collagen fibers and long axis of the meniscus. Radial tears are also known as free edge tears because they involve inner edge of the meniscus. MR images obtained perpendicular to the orientation of radial tear easily demonstrates tear as a small gap or cleft in the meniscus (Cleft sign), which sometimes can be observed to move across the meniscus on successive images (Margin Cleft sign). If the orientation of the imaging plane is in the same direction as a full thickness radial tear, a section through the same plane of the tear will demonstrate the absent meniscus or high signal intensity not representative of meniscus because of volume averaging, which results in the so called Ghost sign. A partial thickness radial tear will demonstrate blunting or abrupt truncation of the apex of the meniscus (truncated triangle sign). The term parrot beak tear has been frequently used to describe a vertical radial tear at the inner edge of the meniscus that curves obliquely across the circumferential collagen fibers.(Fig 10) 


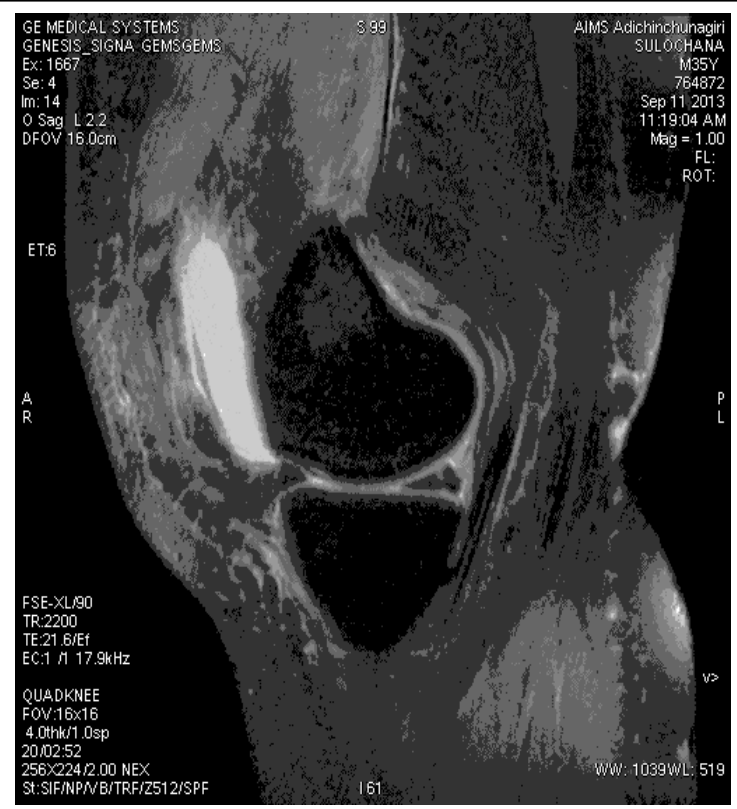

Fig-10 Sagittal images of Proton density fat sat shows vertical tear in the posterior horn of the medial meniscus.

Root tears: They are full thickness radial tears at the central tibial attachment sites of the menisci and are called the root ligaments. A root tear is reportedly difficult tear to diagnose because meniscal tissue is noted only on one side of the tear. The diagnosis is easier to make medially because of the close anatomic relationship between posterior horn of the meniscus and the tibial attachment of the PCL.

Normally, on 3-mm sagittal images, the meniscus should be seen on the image medial to the PCL attachment; otherwise, a root tear is suspected and the coronal images can confirm. Lateral meniscal root tears are diagnosed when posterior horn of the lateral meniscus does not cover the most medial aspect of posterior lateral tibial plateau on at least one coronal image.(Fig 11)

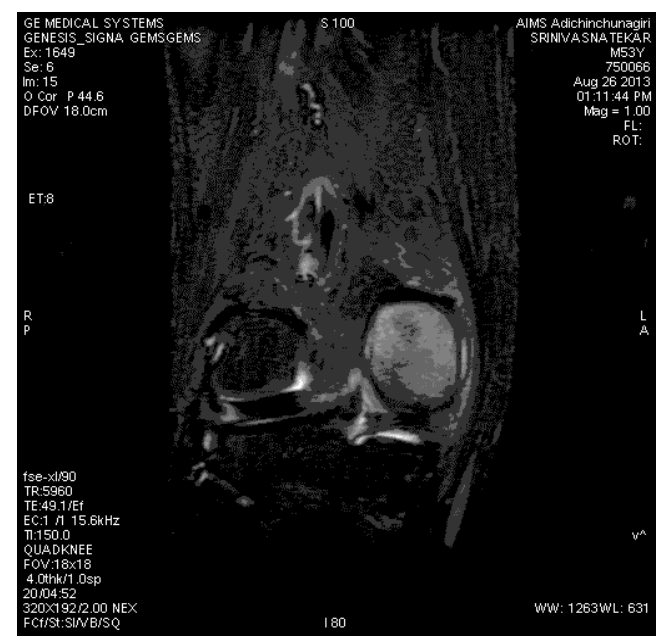

Fig-11 Coronal STIR image shows posterior root tear of the medial meniscus.

Bucket-handle tears: These are displaced longitudinal tears. The displaced in a fragment often has the appearance of a handle, and the remaining peripheral segment attached to the tibial resembles a bucket - hence the name. Confirmation of this tear is mandatory and can be made by identification of displaced fragment. The most reliable sign is the finding of a displaced fragment of meniscus in the intercondylar notch, which can be better seen on coronal images. This sign is associated with a truncated or shortened meniscus on coronal images. On sagittal images, the displaced fragment can be seen lying anterior and parallel to the PCL. This is known as the double PCL sign. These tears may also result in the flipped meniscus sign, double delta sign, or double anterior horn sign, in which the displaced meniscal segment of the posterior horn is located anterior to, on top of, or posterior to the anterior horn. Helpful clues in these cases include non visualization of the posterior horn of the meniscus, the appearance of a larger anterior horn than posterior horn, or an anterior horn taller than $6 \mathrm{~mm}$. Fig -12) 


\section{JMSCR Vol||3||Issue||12||Page 8590-8631||December}
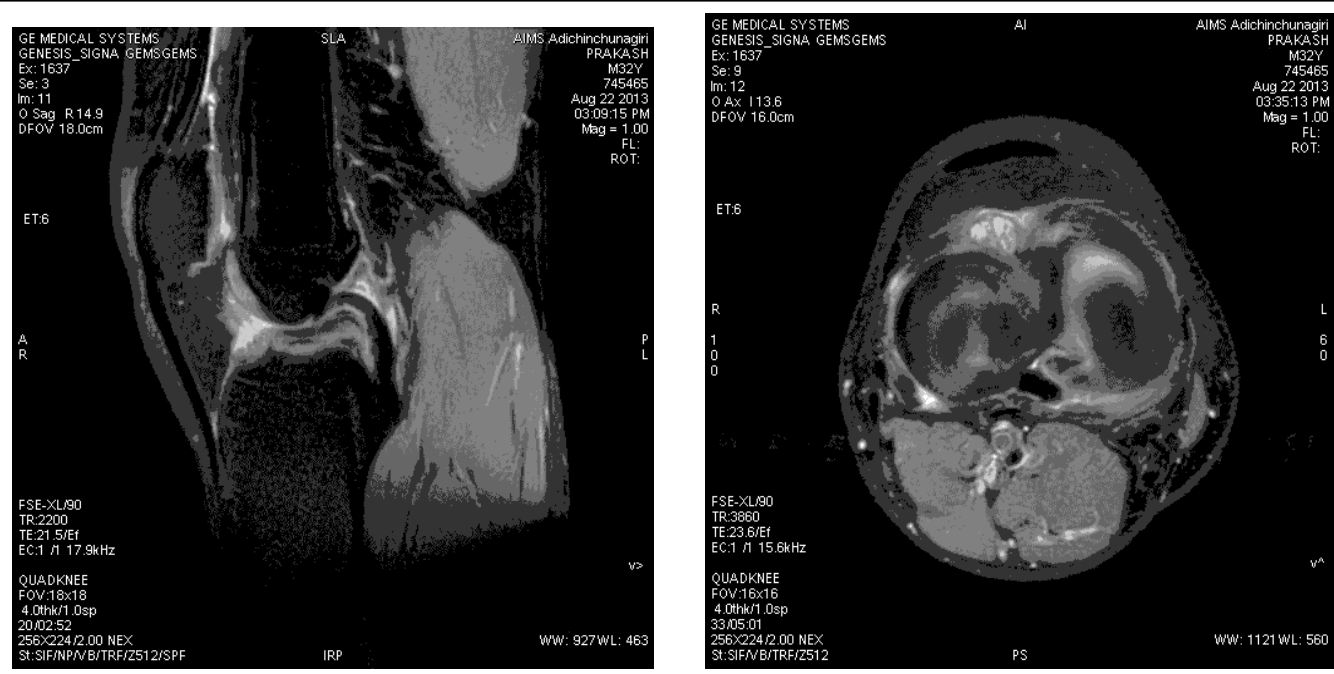

A Fig-12 B

Sagittal images (A) and axial images (B) of proton density fat saturation shows bucket handle tear. A para meniscal cyst is seen anterior to the lateral meniscus $(B)$

Flap Tears: A flap tear or a displaced flap tear is a term that is used often to describe a short- segment, horizontal meniscal tear with fragments either displaced into the notch or into the superior or inferior gutters. Flap tears are the most common type of meniscal tears and may develop following only minimal meniscal trauma of the degenerative meniscus from chronic shear stress. They involve the inner one third to one half of the meniscus with a superior or inferior leaf extension that creates the flap.

3. Complex tears: They either have two or more tear configurations or are not categorized easily into a certain type of tear.

MRI grading of meniscal tears and degenerations:

A grading system based on the signal abnormality correlated with histopathological abnormalities has been developed to understand the significance of increased signal intensity patterns in meniscal abnormalitie.

Grade I: Non-articular focal or globular intrasubstance increased signal intensity in the nonarticular portion of the meniscus.

Grade II: Horizontal linear intrasubstance increased signal intensity usually extends from the capsular periphery but does not involve an articular surface.

Grade III: Region of abnormal signal intensity extends or communicates to at least one of the articular surfaces.
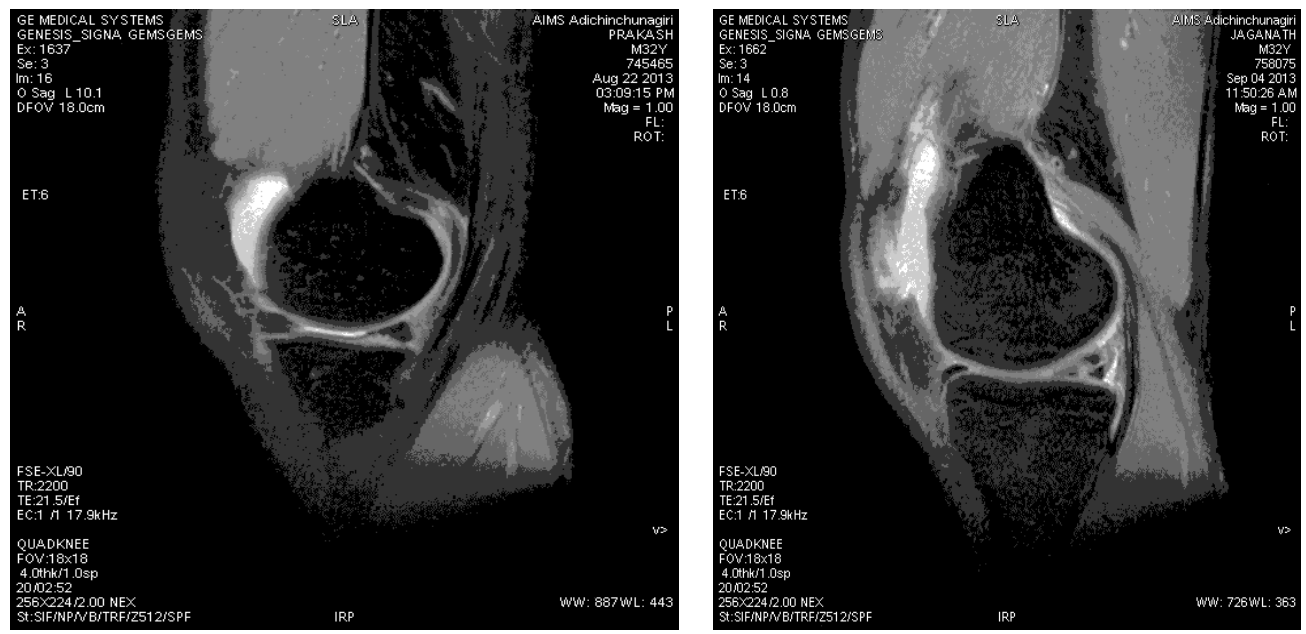

A Fig-13 B A-Linear high signal intensity that intersects the inferior articular surface representing a grade 3 lesion. B-Complex grade 3 tears extending to both the superior and inferior articular surfaces 


\section{MENISCAL CYST}

A meniscal cyst is a fluid-filled structure adjacent to a meniscus, which appears as intermediate signal intensity mass on T1weighted images and hyperintense on T2-weighted images, sometimes lobulated or septated. Meniscal cysts are strongly associated with a horizontal cleavage tear of the meniscus and often a connecting neck between the tear and the cyst can be visualized. The cysts may become fairly large, which is thought to be caused by a ball valve mechanism. They can become symptomatic usually on the medial side, since they may impress on the medial collateral ligament.

\section{MENISOCAPSULAR SEPERATION}

Meniscocapsular separation occurs when the meniscus detaches from the capsular attachments, which is more common medially and usually is associated with other injuries. Meniscocapsular separation is evaluated best on coronal and sagittal T1- or proton density weighted sequences for anatomy, and fat-saturated T2-weighted or STIR sequences for pathology. Signs that have been described in meniscocapsular separation include displacement of the meniscus relative to the tibial margin, extension of the tear into the superior or inferior corner of the peripheral meniscus, and an irregular outer margin of the meniscus body on coronal images. Additional signs include increased distance between the meniscus and the MCL, or fluid between the meniscus and the MCL. Overall, the presence of perimeniscal fluid and an irregular meniscal outline are the best predictors of meniscocapsular separation.

\section{IV) EXTENSOR MECHANISM AND PATELLAR TENDON}

The extensor mechanism of the knee consists of the quadriceps muscle group, quadriceps tendon, patella, patellar retinaculum, patellar ligament, and adjacent soft tissues. Injuries to the extensor mechanism are common and consist of chronic degenerative injuries, overuse injuries, and acute trauma. Overuse injuries typically are seen in high performance athletes, most commonly in sports requiring long distance running or jumping. Cumulative micro trauma produced by repetitive knee flexion and extension results in focal inflammation.

Acute traumatic injuries occur secondary to direct blunt trauma or excessive tension applied to the extensor mechanism through quadriceps contraction. Patellar fractures are the most common traumatic injury of the knee extensor mechanism.

\section{Patellar Tendinopathy}

Patellar injuries can result from chronic overuse injuries or as a result of acute trauma. Patellar tendonosis, or 'jumper's knee' results from chronic overload on the patellar tendon because of strain from the quadriceps muscle group. Chronic overload results in microtears and focal degeneration, which is characterized histologically by pseudocyst formation, increased fibrocartilage, and myxomatous and hyaline metaplasia. Patellar tendonosis frequently is seen in participants in sports associated with jumping and is associated with mal-alignment.

MRI findings consist of focal thickening of the proximal patellar tendon, with increased signal on T1-weighted and T2-weighted images. Edema in the paratenon, infrapatellar fat pad, and subcutaneous adipose tissue is associated with acute patellar tendonitis, which is defined as symptoms of less than 2-week duration and patellar tendon tears. Acute patellar tendonitis is associated with increased intrasubstance signal on T1-weighted and T2-weighted images without tendon thickening.

\section{Patellar tendon tears}

They may be partial or complete, and most frequently occur in the proximal inferior patellar tendon.A patellar sleeve fracture represents a cartilaginous avulsion from the lower pole of the patella and is often associated with patellar tendon tears. With complete inferior patellar tendon tears, the patella retracts proximally as a result of the pull of the superior patellar tendon.

MRI shows discontinuity of the tendon, demonstrated on fluid-sensitive sagittal T2-weighted and STIR images. MRI findings in partial patellar tendon tears can resemble acute patellar tendonitis.

Correlation with a history of acute trauma is useful for specific diagnosis. 


\section{Quadriceps tendon tear}

A tear of the quadriceps tendon is the second most common acute injury to the extensor mechanism.Injury to the quadriceps mechanism (rectus femoris, vastus medialis, vastus intermedius, vastus lateralis, and quadriceps tendon) may be the result of acute trauma caused by rapid deceleration, such as running when the foot is planted, or it may be caused by chronic microtrauma. In elderly patients or patients who have gout, diabetes, connective tissue diseases, or other systemic conditions, minor trauma may result in quadriceps injury.

MRI is useful in differentiating complete from partial quadriceps tendon tears. With complete tears, discontinuity of the tendon with associated retraction of the proximal remnant usually is visualized best using T2-weighted images. Often, an associated hematoma is seen with heterogeneous signal intensity on T1-weighted images.

Myotendinous strain:

Myotendinous strain is a common injury in athletes. The most commonly injured muscles are fusiform muscles that cross two joints. In these muscles, high tensile stress develops when the muscle is forced to lengthen during active contraction, a process termed eccentric contraction.Because the rectus femoris muscle is the only component of the quadriceps muscle group that crosses two joints, it is the most common site for myotendinous strain injuries in the knee extensor mechanism.

MRI findings of strain injuries consist of focal edema centered on the central tendon of the rectus femoris. The presence of a focal hematoma is indicative of a partial myotendinous tear. Complete myotendinous tears often are recognized clinically, and these patients less frequently undergo MRI.

\section{V) OSSEOUS AND OSTEOCHONDRAL LESIONS}

\section{Bone bruise or marrow edema pattern}

Bone bruises or marrow edema may be identified with numerous conditions, including trauma, infection, and osteoporosis. Bone bruises related to trauma may be caused by a direct blow, articular compression forces, or avulsion injuries. The extent of marrow edema tends to be more dramatic with compression or direct trauma, compared with avulsion injuries. Typically, bone bruises are not visible on radiographs. However, these injuries are detected easily on MR images using T1- weighted sequences and either short T1 inversion recovery (STIR) or fat-suppressed T2-weighted sequences. Specific edema patterns are also useful in predicting the mechanism of injury and the associated ligament, tendon, or meniscal involvement.

\section{Tibial plateau fractures}

Subtle tibial plateau fractures are overlooked easily on radiographs. The only finding may be a lipohemarthrosis, which can be detected on the cross table lateral radiograph. Displaced tibial plateau fractures are detected easily on radiographs. However, further imaging with CT or MR imaging is required to evaluate fragment position and associated soft-tissue injuries. CT is adequate for the evaluation of fracture position, articular step-off, and separation of fragments. MR imaging, especially with subtle fractures, can assess the bony injury and associated ligament and meniscal injury more easily.

\section{Segond and reverse Segond fractures}

The Segond fracture is a small, avulsion fracture proximal and posterior to the insertion of the iliotibial band on Gerdy's tubercle. The avulsion may occur with internal rotation and the knee flexed, or with internal rotation and varus stress. The fracture is caused by avulsion of the meniscotibial portion of the middle third of the lateral capsular ligament, with possible associated fractures of the fibular head or Gerdy's tubercle. In the case of severe varus stress, such as in a motor vehicle accident, the entire lateral ligament complex may be disrupted. Segond fractures have a high incidence of anterior cruciate ligament tears and meniscal injury. MR imaging is ideal for the detection of osseous, meniscal, and ligament injuries.

The reverse Segond fracture, much less common, is a medial avulsion of the deep medial collateral ligament attachment on the tibia. The mechanism of injury is felt to be valgus stress with external rotation and is associated with disruption of the posterior cruciate ligament and peripheral medial meniscus tears. When this avulsion is detected on radiographs, MR imaging should be performed to evaluate the associated ligament and meniscal injuries. 


\section{Osteochondral lesions}

Osteochondral lesions in the knee may be acute or chronic. Acute lesions result from impaction, or rotational or shearing forces. The fracture may involve only cartilage, or cartilage and underlying bone. The fracture line is parallel to the joint line, unlike more conventional fractures that enter the joint vertically or obliquely. Fractures may be impacted, elevated partially or displaced completely and free in the joint. When imaging osteochondral lesions, radiographs may be useful, but detection and appropriate classification are accomplished more effectively with MR imaging using T1- and T2-weighted or STIR sequences. The axial and sagittal planes are most useful for femoral lesions. Lesions that are minimal (softening, fibrillation, or fissuring) show abnormal signal intensity without elevation or separation. MR signs of unstable lesions include a high signal intensity line between the native bone and the osteochondral fragment, focal defects, articular fracture, and adjacent subchondral cysts.

Spontaneous osteonecrosis of the knee:

Spontaneous osteonecrosis of the knee (SONK): Spontaneous osteonecrosis of the knee was considered classically a condition of vascular insufficiency, leading to bone infarction of the weight bearing surface of the femoral condyle. The condition was seen in older adults. The condition almost always was unilateral and involved most commonly the medial femoral condyle. growing base of knowledge in the literature suggests that a subchondral insufficiency fracture leads to focal osteonecrosis. Similar features have been described in the medial tibial plateau as well.

\section{ARTHROSCOPY}

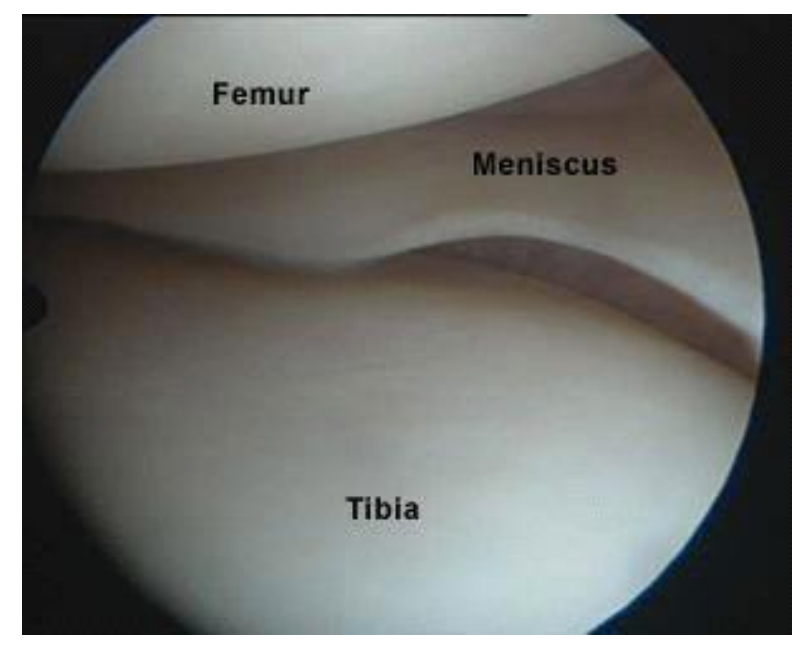

Fig-14 Arthroscopic view of normal study

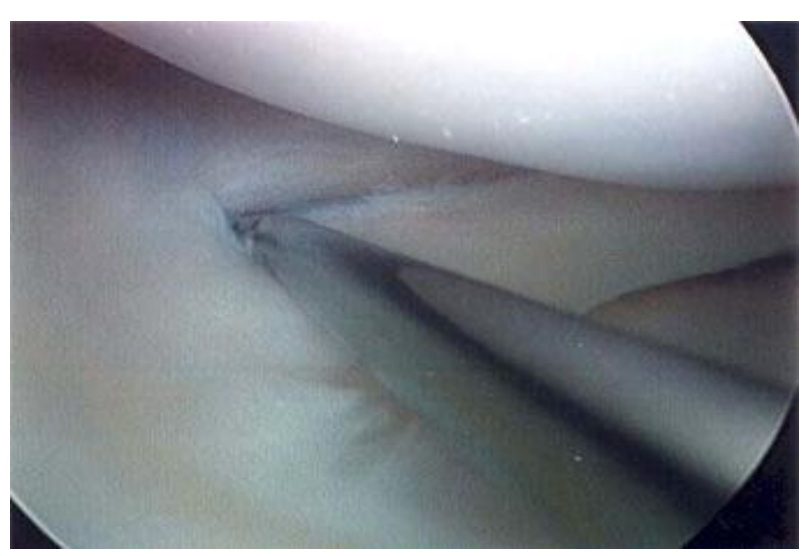

Fig-15 Arthroscopic view of horizontal tear 


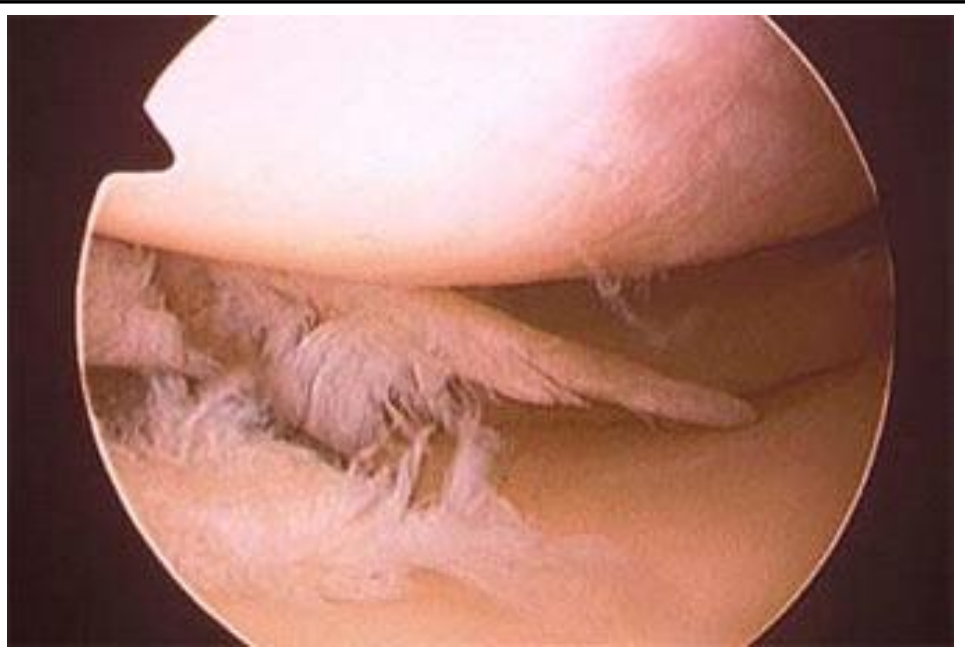

Fig-16 Arthroscopic view of complex tear

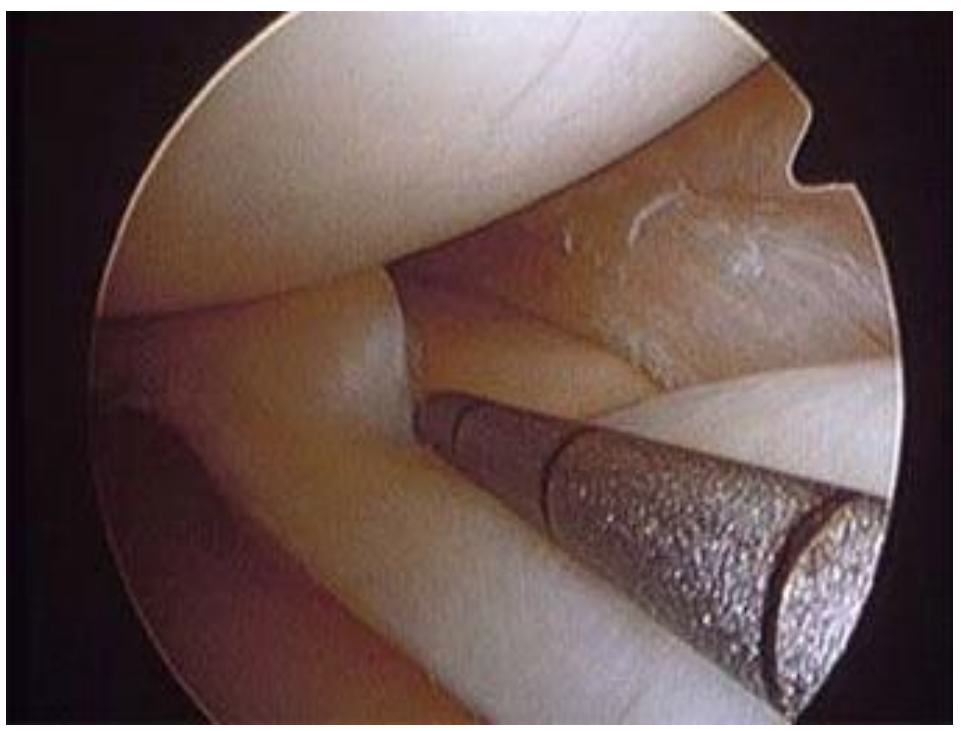

Fig-17 Arthroscopic view of bucket handle tear

\section{METHODOLOGY}

\section{Source of Data}

Cases referred from the Department of Orthopaedics of Rajarajeswari mediacal college and hospital, were included in the study. Study span is about 18 months.

\section{Method of Collection of Data}

Duration of study: November 2012 - November 2014.

Sample size: 50 cases.

Methods: All patients were subjected to MR imaging and followed by arthroscopy.

\section{Inclusion criteria:}

Patients with history of knee joint injury referred from the department of orthopaedics for suspected internal derangement of knee joint and who underwent both MRI and Arthroscopy

Exclusion criteria: 
- $\quad$ Patients with ferromagnetic implants, pacemakers, and aneurysm clips.

- Patients with major injuries like liver / splenic rupture and flail chest and patients with unstable vital parameters especially in the setting of trauma

- $\quad$ Patients with knee joint neoplasm

Imaging Protocols: Patients will be subjected to MRI within 4 days of clinical evaluation according to the following protocols:

Equipment: Signa contour GE 1.5 Tesla. Whole- body MR scanner with knee coil.

\title{
Protocol :
}

- $\quad$ T1 and PD FS weighted sequences in sagittal and coronal planes.

- $\quad$ T2- weighted in axial, coronal and sagittal planes.

- $\quad$ Fat suppressed T2 or STIR sequences wherever indicated.

\section{Interpretation of MRI Data}

evaluated for:

\author{
Anterior Cruciate Ligament tear: Present/Absent \\ Partial/Complete \\ Posterior Cruciate Ligament tear: Present/Absent, \\ Partial/Complete \\ Medial Meniscal Tear: Present/Absent \\ Location and Type \\ Lateral Meniscal Tear: Present/Absent \\ Location and Type
}

Interpretation of Arthroscopy Data: was carried out in OT by orthopedic surgeon in selected cases where indicated for diagnostic or therapeutic purposes.

\section{- $\quad$ Equipment}

- Interpretation of Arthroscopic Data

Study will be evaluated for:

Anterior Cruciate Ligament tear: Present/Absent

Partial/Complete

Posterior Cruciate Ligament tear: Present/Absent,

Partial/Complete

Medial Meniscal Tear: Present/Absent

Location and Type

Lateral Meniscal Tear: Present/Absent

Location and Type 


\section{RESULTS}

\section{CASE-1}

1. Name $\quad$ : Mr. Chaluvaraj

2. Age $: 36 \mathrm{Y}$

3. Sex

$$
\text { : M }
$$

4. Involvement of joint : Left

5. Investigation Results

\section{MRI Findings}

a) Anterior Cruciate Ligament tear : Absent

b) Posterior Cruciate Ligament tear : : Absent

c) Medial Meniscal Tear $\quad$ : $\quad$ Tear of body and posterior horn of medial meniscus.

d) Lateral Meniscal Tear $\quad$ : Absent

Arthroscopic findings $\quad$ : $\quad$ Tear of body and posterior horn of medial meniscus

Result: MRI finding correlating with arthroscopy findings.

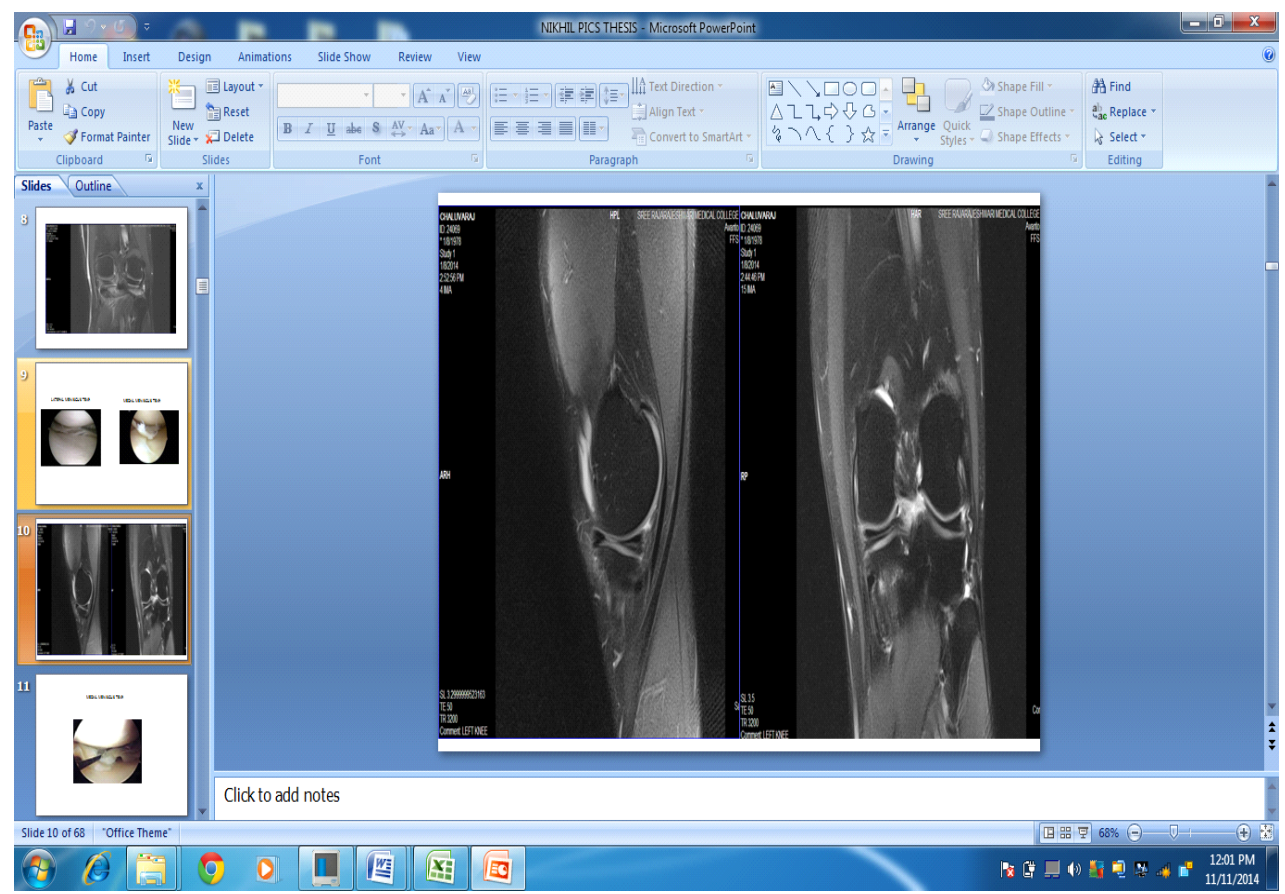

Fig-18 A and B Sagittal images of PD FS (A) and COR(B), showing tear of body and posterior horn of medial meniscus. 


\section{JMSCR Vol||3||Issue||12||Page 8590-8631||December}

CASE-2

1. Name : Mr. Mahadev

2. Age $: 28 \mathrm{Y}$

3. Sex : M

4. Involvement of joint : L

5. Investigation Results

MRI Findings

a) Anterior Cruciate Ligament tear : complete tear

b) Posterior Cruciate Ligament tear : : Absent

c) Medial Meniscal Tear $\quad: \quad$ Absent

d) Lateral Meniscal Tear $\quad$ : Absent

Arthroscopic findings : : Complete ACL tear.

Result: MRI finding correlating with arthroscopy findings.

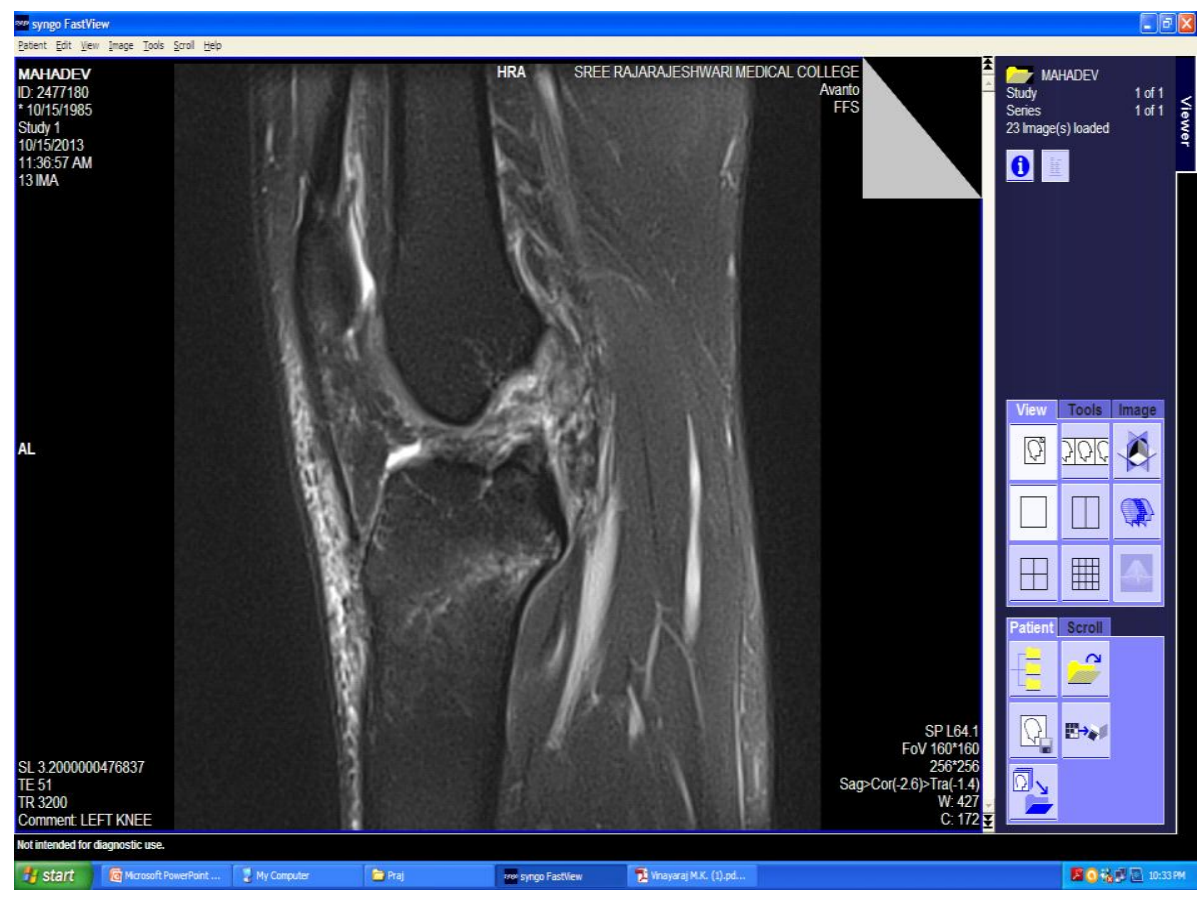

Fig- 19 Sagittal images of PD FS showing complete tear of ACL.

\section{CASE-3}

1. Name $\quad$ : Mr. Vishnu ramesh

2. Age

: $28 \mathrm{Y}$ 


\section{JMSCR Vol||3||Issue||12||Page 8590-8631||December}

3. Sex

: M

4. Involvement of joint : $\mathrm{R}$

5. Investigation Results

\section{MRI Findings}

a) Anterior Cruciate Ligament tear : Absent

b) Posterior Cruciate Ligament tear : : $\quad$ Complete mid substance tear

c) Medial Meniscal Tear $\quad$ : Absent

d) Lateral Meniscal Tear $\quad$ : Absent

Arthroscopic findings $\quad$ : Complete mid substance PCL tear

Result: MRI finding correlating with arthroscopy findings.

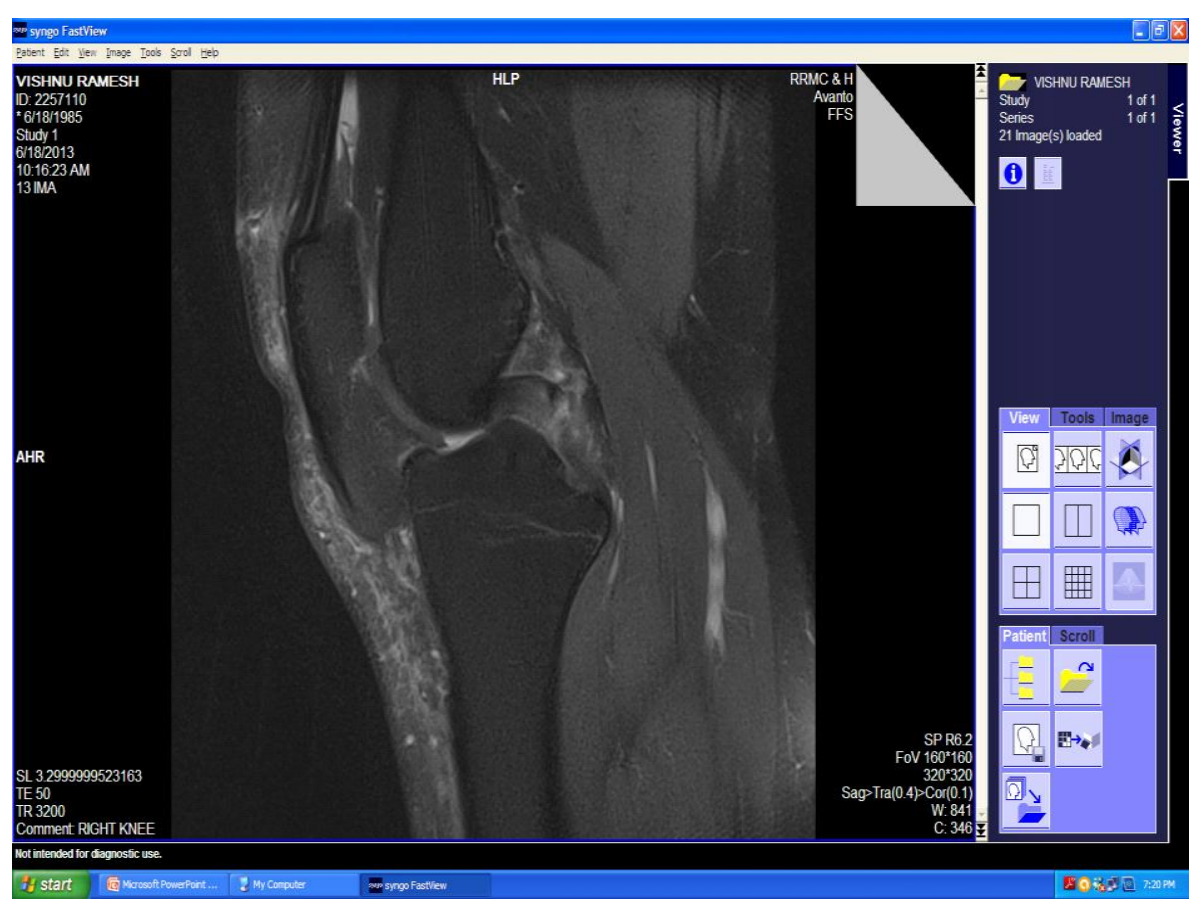

Fig- 20 Sagittal image of PD FS showing complete mid substance PCL tear.

\section{CASE-4}

1. Name $\quad$ Mr. Chandrashekar

2. Age $: 29 \mathrm{Y}$

3. Sex :M

4. Involvement of joint : $\mathrm{R}$ 
5. Investigation Results

\section{MRI Findings}

a) Anterior Cruciate Ligament tear : Absent

b) Posterior Cruciate Ligament tear $\quad$ : Absent

c) Medial Meniscal Tear $\quad: \quad$ Absent

d) Lateral Meniscal Tear $\quad$ : $\quad$ Tear of body and posterior horn of lateral meniscus

Arthroscopic findings $\quad: \quad$ Tear of body and posterior horn of lateral meniscus

Result: MRI finding correlating with arthroscopy findings.
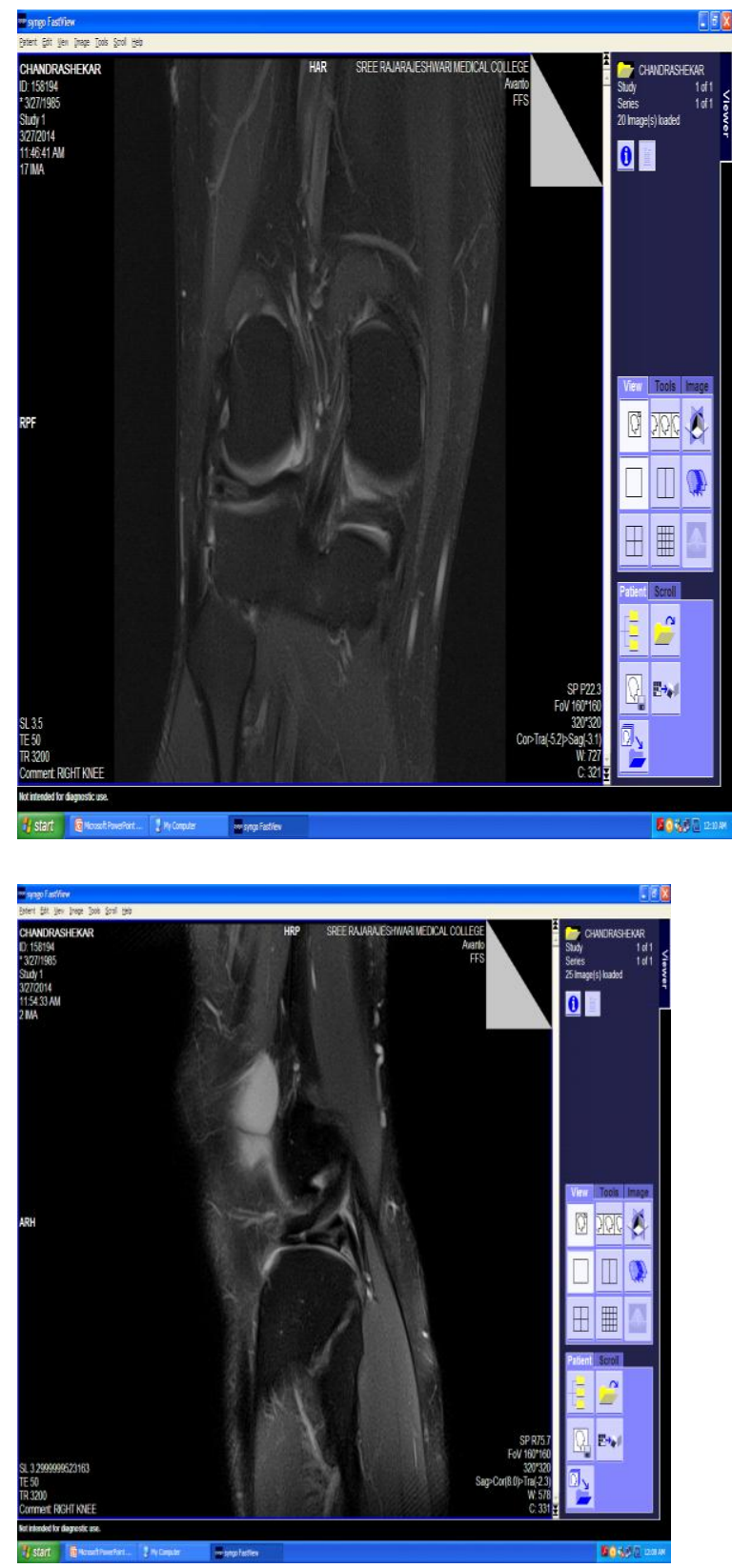

Fig -21 (A) and (B) Coronal images of PD FS (A) and Sag PDFS (B) showing tear of body and posterior horn of lateral meniscus. 


\section{OBSERVATIONS}

Table 1: Association between Anterior Cruciate Ligament involvement and Diagnostic Modality $(\mathrm{N}=50)$

\begin{tabular}{|l|l|l|l|}
\hline \multirow{2}{*}{$\begin{array}{l}\text { ACL } \\
\text { Involvement }\end{array}$} & MRI & Arthroscopy & \multirow{2}{*}{ P Value } \\
\cline { 2 - 3 } & No. (\%) & No. (\%) & \\
\hline Yes & $33(\mathbf{6 6 . 0})$ & $18(\mathbf{3 6 . 0})$ & \multirow{2}{*}{$\mathbf{0 . 0 0 3}$} \\
\hline \multicolumn{1}{|c|}{ No } & $17(\mathbf{3 4 . 0})$ & $32(\mathbf{6 4 . 0})$ & \\
\hline
\end{tabular}

Table 1: Sensitivity, Specificity and Predictive Accuracy of MRI in the Diagnosis of Anterior Cruciate Ligament Injuries Compared to Arthroscopy $(\mathbf{N}=\mathbf{5 0})$

\begin{tabular}{|c|c|c|c|}
\hline \multicolumn{2}{|l|}{ ACL Injuries } & \multicolumn{2}{|c|}{ Arthroscopy Findings } \\
\hline & & Yes & No \\
\hline \multirow{2}{*}{ MRI Findings } & Yes & 17 & 16 \\
\hline & No & 1 & 16 \\
\hline \multicolumn{4}{|c|}{ Sensitivity $=94.4 \%$} \\
\hline \multicolumn{4}{|c|}{ Specificity $=50.0 \%$} \\
\hline \multicolumn{4}{|c|}{ Positive Predictive Value $=51.5 \%$} \\
\hline \multicolumn{4}{|c|}{ Negative Predictive Value $=94.1 \%$} \\
\hline
\end{tabular}

Table 2: Association between Posterior Cruciate Ligament involvement and Diagnostic Modality $(\mathrm{N}=50)$

\begin{tabular}{|l|l|l|l|}
\hline \multirow{2}{*}{$\begin{array}{l}\text { PCL } \\
\text { Involvement }\end{array}$} & MRI & Arthroscopy & \multirow{2}{*}{ P Value } \\
\cline { 2 - 3 } & No. (\%) & No. (\%) & \\
\hline Yes & $6(\mathbf{1 2 . 0})$ & $6(\mathbf{1 2 . 0})$ & \multirow{2}{*}{1.000} \\
\hline \multicolumn{1}{|c|}{ No } & $44(\mathbf{8 8 . 0})$ & $44(\mathbf{8 8 . 0})$ & \\
\hline
\end{tabular}

Table 2: Sensitivity, Specificity and Predictive Accuracy of MRI in the Diagnosis of Posterior Cruciate Ligament Injuries Compared to Arthroscopy $(\mathbf{N}=50)$

\begin{tabular}{|c|c|c|c|}
\hline \multicolumn{2}{|l|}{ PCL Injuries } & \multicolumn{2}{|c|}{ Arthroscopy Findings } \\
\hline & & Yes & No \\
\hline \multirow{2}{*}{ MRI Findings } & Yes & 6 & 0 \\
\hline & No & 0 & 44 \\
\hline \multicolumn{4}{|c|}{ Sensitivity $=100.0 \%$} \\
\hline \multicolumn{4}{|c|}{ Specificity $=100.0 \%$} \\
\hline \multicolumn{4}{|c|}{ Positive Predictive Value $=100.0 \%$} \\
\hline \multicolumn{4}{|c|}{ Negative Predictive Value $=100.0 \%$} \\
\hline
\end{tabular}


Table 3: Association between Medial Menisci involvement and Diagnostic Modality $(\mathbf{N}=50)$

\begin{tabular}{|l|l|l|l|}
\hline \multirow{2}{*}{$\begin{array}{l}\text { MM } \\
\text { Involvement }\end{array}$} & MRI & Arthroscopy & \multirow{2}{*}{ P Value } \\
\cline { 2 - 3 } & No. (\%) & No. (\%) & \\
\hline Yes & $26(\mathbf{5 2 . 0})$ & $25(\mathbf{5 0 . 0})$ & \multirow{2}{*}{0.841} \\
\hline \multicolumn{1}{|c|}{ No } & $24(\mathbf{4 8 . 0})$ & $25(\mathbf{5 0 . 0})$ & \\
\hline
\end{tabular}

Table 3: Sensitivity, Specificity and Predictive Accuracy of MRI in the Diagnosis of Medial Menisci Injuries Compared to Arthroscopy $(\mathbf{N}=\mathbf{5 0})$

\begin{tabular}{|c|c|c|c|}
\hline \multicolumn{2}{|l|}{ MM Injuries } & \multicolumn{2}{|c|}{ Arthroscopy Findings } \\
\hline & & Yes & No \\
\hline \multirow{2}{*}{ MRI Findings } & Yes & 25 & 1 \\
\hline & No & 0 & 24 \\
\hline \multicolumn{4}{|c|}{ Sensitivity $=100.0 \%$} \\
\hline \multicolumn{4}{|c|}{ Specificity $=96.0 \%$} \\
\hline \multicolumn{4}{|c|}{ Positive Predictive Value $=96.2 \%$} \\
\hline \multicolumn{4}{|c|}{ Negative Predictive Value $=100.0 \%$} \\
\hline
\end{tabular}

Table 4: Association between Lateral Menisci involvement and Diagnostic Modality $(\mathrm{N}=50)$

\begin{tabular}{|l|l|l|l|}
\hline \multirow{2}{*}{$\begin{array}{l}\text { LM } \\
\text { Involvement }\end{array}$} & MRI & Arthroscopy & \multirow{2}{*}{ P Value } \\
\cline { 2 - 3 } & No. (\%) & No. (\%) & \\
\hline Yes & $19(\mathbf{3 8 . 0})$ & $18(\mathbf{3 6 . 0})$ & \multirow{2}{*}{0.836} \\
\hline \multicolumn{1}{|c|}{ No } & $31(\mathbf{6 2 . 0})$ & $32(\mathbf{6 4 . 0})$ & \\
\hline
\end{tabular}

Table 4: Sensitivity, Specificity and Predictive Accuracy of MRI in the Diagnosis of Lateral Menisci Injuries Compared to Arthroscopy (N = 50)

\begin{tabular}{|c|c|c|c|}
\hline \multicolumn{2}{|l|}{ LM Injuries } & \multicolumn{2}{|c|}{ Arthroscopy Findings } \\
\hline & & Yes & No \\
\hline \multirow{2}{*}{ MRI Findings } & Yes & 18 & 1 \\
\hline & No & 0 & 31 \\
\hline \multicolumn{4}{|c|}{ Sensitivity $=100.0 \%$} \\
\hline \multicolumn{4}{|c|}{ Specificity $=96.9 \%$} \\
\hline \multicolumn{4}{|c|}{ Positive Predictive Value $=94.7 \%$} \\
\hline \multicolumn{4}{|c|}{ Negative Predictive Value $=100.0 \%$} \\
\hline
\end{tabular}


Table 5: Association between Medial Collateral Ligament involvement and Diagnostic Modality $(\mathrm{N}=50)$

\begin{tabular}{|l|l|l|l|}
\hline \multirow{2}{*}{$\begin{array}{l}\text { MCL } \\
\text { Involvement }\end{array}$} & MRI & Arthroscopy & \multirow{2}{*}{ P Value } \\
\cline { 2 - 3 } & \multicolumn{1}{|c|}{ No. (\%) } & \multicolumn{1}{|c|}{ No. (\%) } & \\
\hline Yes & $0(\mathbf{0 . 0})$ & $0(\mathbf{0 . 0})$ & \multirow{2}{*}{ NA } \\
\hline \multicolumn{1}{|c|}{ No } & $50(\mathbf{1 0 0 . 0})$ & $50(\mathbf{1 0 0 . 0})$ & \\
\hline
\end{tabular}

Table 5: Sensitivity, Specificity and Predictive Accuracy of MRI in the Diagnosis of Medial Collateral Ligament Injuries Compared to Arthroscopy $(\mathrm{N}=\mathbf{5 0})$

\begin{tabular}{|c|c|c|c|}
\hline \multicolumn{2}{|l|}{ MCL Injuries } & \multicolumn{2}{|c|}{ Arthroscopy Findings } \\
\hline & & Yes & No \\
\hline \multirow{2}{*}{ MRI Findings } & Yes & 0 & 0 \\
\hline & No & 0 & 50 \\
\hline \multicolumn{4}{|l|}{ Sensitivity $=$ NA } \\
\hline \multicolumn{4}{|c|}{ Specificity $=100.0 \%$} \\
\hline \multicolumn{4}{|c|}{ Positive Predictive Value $=$ NA } \\
\hline \multicolumn{4}{|c|}{ Negative Predictive Value $=100.0 \%$} \\
\hline
\end{tabular}

Table 6: Association between Lateral Collateral Ligament involvement and Diagnostic Modality $(\mathrm{N}=\mathbf{5 0})$

\begin{tabular}{|l|l|l|l|}
\hline \multirow{2}{*}{$\begin{array}{l}\text { LCL } \\
\text { Involvement }\end{array}$} & MRI & Arthroscopy & \multirow{2}{*}{ P Value } \\
\cline { 2 - 3 } & No. (\%) & \multicolumn{1}{|c|}{ No. (\%) } & \\
\hline Yes & $0(\mathbf{0 . 0})$ & $0(\mathbf{0 . 0})$ & \multirow{2}{*}{ NA } \\
\hline \multicolumn{1}{|c|}{ No } & $50(\mathbf{( 1 0 0 . 0 )}$ & $50(\mathbf{1 0 0 . 0})$ & \\
\hline
\end{tabular}

Table 6: Sensitivity, Specificity and Predictive Accuracy of MRI in the Diagnosis of Lateral Collateral Ligament Injuries Compared to Arthroscopy $(\mathrm{N}=\mathbf{5 0})$

\begin{tabular}{|c|c|c|c|}
\hline \multicolumn{2}{|l|}{ LCL Injuries } & \multicolumn{2}{|c|}{ Arthroscopy Findings } \\
\hline & & Yes & No \\
\hline \multirow{2}{*}{ MRI Findings } & Yes & 0 & 0 \\
\hline & No & 0 & 50 \\
\hline \multicolumn{4}{|c|}{ Sensitivity $=$ NA } \\
\hline \multicolumn{4}{|c|}{ Specificity $=100.0 \%$} \\
\hline \multicolumn{4}{|c|}{ Positive Predictive Value $=$ NA } \\
\hline \multicolumn{4}{|c|}{ Negative Predictive Value $=100.0 \%$} \\
\hline
\end{tabular}


Table 7: Association between Osteo-Chondral Defects and Diagnostic Modality (N = 50)

\begin{tabular}{|l|l|l|l|}
\hline \multirow{2}{*}{$\begin{array}{l}\text { OCD } \\
\text { Involvement }\end{array}$} & MRI & Arthroscopy & \multirow{2}{*}{ P Value } \\
\cline { 2 - 3 } & \multicolumn{1}{|c|}{ No. (\%) } & No. (\%) & \\
\hline Yes & $2 \mathbf{( 4 . 0 )}$ & $2(\mathbf{4 . 0})$ & \multirow{2}{*}{1.000} \\
\hline \multicolumn{1}{|c|}{ No } & $48(\mathbf{9 6 . 0 )}$ & $48(\mathbf{9 6 . 0 )}$ & \\
\hline
\end{tabular}

Table 7: Sensitivity, Specificity and Predictive Accuracy of MRI in the Diagnosis of Osteo-Chondral Defects Compared to Arthroscopy (N = 50)

\begin{tabular}{|c|c|c|c|}
\hline \multicolumn{2}{|l|}{ OCD Injuries } & \multicolumn{2}{|c|}{ Arthroscopy Findings } \\
\hline & & Yes & No \\
\hline \multirow{2}{*}{ MRI Findings } & Yes & 1 & 0 \\
\hline & No & 0 & 48 \\
\hline \multicolumn{4}{|c|}{ Sensitivity $=100.0 \%$} \\
\hline \multicolumn{4}{|c|}{ Specificity $=100.0 \%$} \\
\hline \multicolumn{4}{|c|}{ Positive Predictive Value $=100.0 \%$} \\
\hline \multicolumn{4}{|c|}{ Negative Predictive Value $=100.0 \%$} \\
\hline
\end{tabular}

Table 1: Distribution of patients according to their Age Group ( $\mathbf{N}=\mathbf{5 0})$

\begin{tabular}{|c|c|c|}
\hline Age (in Years) & No. & Percent \\
\hline$\leq 25$ & 8 & 16.0 \\
\hline $26-35$ & 22 & 44.0 \\
\hline $36-45$ & 14 & 28.0 \\
\hline$>45$ & 6 & 12.0 \\
\hline Mean \pm SD & \multicolumn{2}{|c|}{$33.5 \pm 10.1$} \\
\hline Range & \multicolumn{2}{|l|}{$16-70$} \\
\hline
\end{tabular}

Table 2: Distribution of patients according to their Gender $(\mathbf{N}=\mathbf{5 0})$

\begin{tabular}{|l|l|l|}
\hline Gender & No. & Percent \\
\hline Male & 39 & $\mathbf{7 8 . 0}$ \\
\hline Female & 11 & $\mathbf{2 2 . 0}$ \\
\hline
\end{tabular}

Table 3: Distribution of patients according to their Presenting Complaints $(\mathbf{N}=50)$

\begin{tabular}{|l|l|l|}
\hline Presenting Complaints & \multicolumn{1}{|c|}{ No. } & Percent \\
\hline Pain & 33 & $\mathbf{6 6 . 0}$ \\
\hline Instability & 10 & $\mathbf{2 0 . 0}$ \\
\hline Both & 7 & $\mathbf{1 4 . 0}$ \\
\hline
\end{tabular}


Table 4: Distribution of patients according to the side involved $(\mathrm{N}=50)$

\begin{tabular}{|c|c|c|}
\hline Side Involved & No. & Percent \\
\hline Left & 30 & 60.0 \\
\hline Right & 20 & 40.0 \\
\hline
\end{tabular}

\section{DISCUSSION}

In the present study of evaluation of internal derangement of knee (and correlation with arthroscopy); we have studied 50 patients of which 39 were male and 11 were female; the mean age at presenetation was in the third decade. Out of the 50 knee joint studied, 30 were left and 20 were right .

Anterior cruciate ligament tears were found to be more commoner (66\%) compared to the posterior cruciate ligament(12\%). Also medial meniscus(52\%) was more commonly injured than lateral meniscus.

\section{Cruciate ligament tears:}

Out of 50 patients, 33 patients (66\%) showed ACL tears. 6 patients (12\%) showed PCL tear. Out of 33 patients of ACL tears, 27 patients (84.2\%) had mid substance tears, 1 patient (3\%) had tear at the femoral attachment and $5(15.1 \%)$ at the tibial attachment were detected, on MRI. In our study, the most common tear location was at mid-substance. On Arthroscopy, out of 50 patients, 18 patients (36\%) showed ACL tears, 6 patients (12\%) showed PCL tear. Out of 18 patients of ACL tears, mid substance tears were seen in 16 patients $(88.8 \%)$, femoral attachment tears in 1 patient (11.1\%) and tibial attachment tears ( 3 patients showed avulsion fracture at the tibial attachment and 1 showed only avulsion) in 4 patients (22.2\%) were detected. Berquist $e t$ al in their study reported midsubstance tears as the most common type (90\%).

In this study we found hyper intensity in the ligament as the most common sign.

In tears of the anterior cruciate ligament, the sensitivity and specificity was found to be $94 \%$ and $50 \%$.

In our study the positive predictive value and negative predictive value was $52 \%$ and $94 \%$ respectively.

Out of 33 patients of ACL tears, 29 patients (87.87\%) had complete tears, 4 patients (12.1\%) had partial tear on MRI. On Arthroscopy, out of 18 patients of ACL tears, complete tear were seen in 16 patients (88.8\%) and partial tear in 2 patients $(11.1 \%)$.

There were 3 false negative cases that had complete tear as seen on arthroscopy, 2 of which were misinterpreted as partial tears, 1 as normal. MR imaging in this patient demonstrated a linear band of intact fibres normally oriented in expected location of the ACL.

In complete tears of the anterior cruciate ligament, the sensitivity, specificity, positive predictive value and negative predictive value were found to be $89.4 \%, 100 \%, 100 \%$ and $73.3 \%$ respectively.

In partial tears of the anterior cruciate ligament, the sensitivity, specificity, positive predictive value and negative predictive value were found to be $100 \%, 94 \%, 25 \%$ and $100 \%$ respectively.

PD FS images showed clearly the signal intensity changes seen with these tears as excellent contrast is provided by normal low signal intensity of ligaments.

6 patient of PCL tear was detected accurately both by MRI and Arthroscopy. The use of MRI to identify PCL tears has proven to be extremely accurate. This might be expected in light of the fact that the PCL is usually very easily visualized as a homogenous, continuous low-signal structure. Several studies have reported sensitivity, specificity, accuracy, positive predictive value and negative predictive value to be $99-100 \%$. In our study too the sensitivity, specificity, accuracy, positive predictive value and negative predictive value was $100 \%$. 
Meniscal tears: Out of 50 patients, 26 (52\%) showed medial meniscal and 19 cases (38\%) showed lateral meniscal tears on MRI which is corresponding with La Prade and colleagues who reported that medial meniscal tears are twice as common as lateral meniscal tears. On arthroscopy 25 (50\%) showed medial meniscal and 18 cases (36\%) showed lateral meniscal tears.

Out of 26 patients with medial meniscal tears, most common tear location was at posterior horn. In our study, we found posterior horn tear in 20 patients (76.9\%), anterior horn tears in 3 patients (11.53\%) and tear in body in 3 patients (11.53\%) on MRI. On arthroscopy posterior horn tears were seen in 22(84.6\%) patients, anterior horn tears in 5 patients $(19.2 \%)$ and tear in the body in 4 patients $(15.3 \%)$.

Out of 19 patients with lateral meniscus tears posterior horn tears was seen most commonly, in 13 patients $(68.42 \%)$ and anterior horn tears were seen in 4 patients $(21.05 \%)$ and tear in the body was seen in 2 patients(10.5\%). On Arthroscopy18 patients with lateral meniscus tears are seen with posterior horn tears were seen in 14 patients (77.7\%)and anterior horn tears in 3 patients $(16.66 \%)$ while tear in the body was seen in 1 patient $(5.55 \%)$.

In our study the sensitivity, specificity, PPV and NPV for detecting medial meniscal tears was $100 \%, 96 \%, 96 \%$ and $100 \%$ respectively which were corresponding to the Fischer et al.

In our study the sensitivity, specificity, PPV and NPV for detecting lateral meniscal tears was $100 \%, 97 \%$, 95\% and $100 \%$ respectively.

The occurrence of the false positive meniscal tears at MRI imaging has been noted earlier. There are explanations for this apparent discrepancy between findings at MR Imaging and arthroscopy.

- Misinterpretation of normal anatomy like Meniscofemoral ligaments etc.

- The presence of intrasubstance tears which are not seen on arthroscopy.

- The operator dependence of Arthroscopy

- $\quad$ The presence of loose bodies

Out of the 26 Medial Meniscal tears, there were three false positive (10.7\%) and one false negative (35.7\%) observations.

In our study, the 2 false positive MRI involved the posterior horn and 1 involved the anterior horn of the meniscus. Out of the 19 Lateral Meniscal tears, there were 4 false positive (21.0\%) and 7 false negative (36.84\%) observations. The exact cause of the false positives in our study was not apparent. It may be attributed to the presence of intra-meniscal tears not communicating with the articular surface of the meniscus or misinterpretation of normal anatomy or operator/ procedure dependant drawback of arthroscopy.

The degeneration and tears of menisci demonstrated as high signal intensity were due to imbibed synovial fluid. These tears are better demonstrated in short TE images like PD FS and GRET2* images. This has been explained by Stoller et al in their study asthe interaction of synovial fluid with large macromolecules in menisci slows rotational rate of protons and shortens $\mathrm{T} 1$ and $\mathrm{T} 2$ values.

In our study, we found that the PDFS and T2* weighted GRE images clearly depicted the tears supported by Rubin $e t$ al.

Out of the 26 patients with medial meniscal tears, 8 had oblique tears, 10 had complex tear, 2 had horizontal tears, 3 had vertical tear, 2 had root tear and 3 had bucket handle tears.

Out of the 19 patients with lateral meniscal tears, 8 had oblique tears, 7 had complex tear, 2 had horizontal tears, 2 had vertical tear and 2 had root tears.

In our study complex and oblique tears were common than other types of tears.

In our study, there was no e/o association between medial meniscus/lateral meniscus tears with the anterior cruciate ligament tears $(\mathrm{P}$ value $>0.05)$. 


\section{SUMMARY}

The present study attempts to determine the role of magnetic resonance imaging in the evaluation of internal derangements of the knee joint. Knee joint being the largest and the most complex weight bearing joint in the body, is subject to damage because of its inherent structural complexity and the various types of forces that it is subjected to.

Magnetic resonance imaging has emerged as the frontline investigation for the evaluation of internal derangements of the knee joint. It is noninvasive, does not involve ionizing radiation and has multiplanar capability with excellent soft tissue delineation. Arthroscopy gives excellent visualization of the interior of the joint, but is invasive and can evaluate only the surface abnormalities.

In the present study, 50 patients with knee joint symptoms, referred for magnetic resonance imaging of the knee joint were evaluated.

Most common presenting symptoms were knee joint pain and joint instability. The common age group to be involved was between 26-35 years. The following patterns of knee injuries were seen:

- Most common injury was ACL tear of which complete tears were more common. Posterior cruciate ligament tears were less common.

- Among the Meniscal injuries, medial Meniscal tears are more common than lateral meniscus.

- The sensitivity, specificity, positive predictive value and negative predictive value of ACL (C+P) tear is $94 \%, 51 \%, 52 \%$ and $94 \%$ respectively.

- The sensitivity, specificity, positive predictive value and negative predictive value of complete ACL tear is $89 \%, 100 \%$, $100 \%$ and $73 \%$ respectively.

- The sensitivity, specificity, positive predictive value and negative predictive value of partial ACL tear is $100 \%, 94 \%$, $25 \%$ and $100 \%$ respectively.

- The sensitivity, specificity, positive predictive value and negative predictive value of meniscus tear (MM+LM) is $100 \%$, $95 \%, 94 \%$ and $100 \%$ respectively.

- The sensitivity, specificity, positive predictive value and negative predictive value of medial meniscus tear is $100 \%$, $96 \%, 96 \%$ and $100 \%$ respectively.

- The sensitivity, specificity, positive predictive value and negative predictive value of lateral meniscus tear is $100 \%$, $97 \%, 95 \%$ and $100 \%$ respectively.

- Posterior horn tears were more common than anterior horn and body in both medial and lateral meniscus.

- Low sensitivity seen in the posterior horn tear of the lateral meniscus.

- The sensitivity, specificity, positive predictive value and negative predictive value of oblique type of meniscal tear is $86 \%, 99 \%, 92 \%$ and $98 \%$ respectively.

- The sensitivity, specificity, positive predictive value and negative predictive value of complex type of meniscal tear is $78 \%, 100 \%, 100 \%$ and $96 \%$ respectively.

- Among 6 cases of vertical tear, 3 cases were true positive, 2 were false negative and 1 was false positive.

- Among 6 cases of bucket handle tear, 3 cases were true positive and 3 were false positive.

- Among 4 cases of radial tear, 3 cases were true positive and 1 was false positive.

- Among 3 cases of horizontal tear, 2 cases were true positive and 1 was false positive.

- MRI is an excellent, noninvasive, radiation free imaging modality with multiplanar capabilities and excellent soft tissue delineation. It can accurately detect, localize and characterize various internal derangements of the knee joint and help in arriving at a correct anatomical diagnosis thereby guiding further management of the patient 
- $\quad$ MRI has high sensitivity and specificity for PCL, LM and MM tears .MRI has high specificity but less sensitive to the ACL tears.MRI has high sensitivity to the complete ACL tears but less sensitive to the partial ACL tears. MRI has high specificity to the partial ACL tears

\section{CONCLUSION}

In conclusion, it can be said that MRI is very accurate in detecting and characterizing internal derangement of knee and the sensitivity and specificity of the MRI in detecting tears of anterior cruciate ligament, posterior cruciate ligament, lateral meniscus and medial meniscus is on par with arthroscopy. In addition MRI also has the advantage of being non-invasive ( compared with arthroscopy) besides being radiation free (compared to CT). Multiplanar imaging capability and excellent soft tissue resolution also add to MRI for a preferred imaging modality.

\section{BIBLIOGRAPHY}

1. DW Polly, JJ Callaghan, RA Sikes, JM McCabe, K McMahon and CG Savory. The Accuracy of Selective Magnetic Resonance Imaging Compared with the Findings of Arthroscopy of the Knee. Am J Bone Joint Surg 1988; 70: $192-198$.

2. Vaz CES, Camargo OP de, Santana PJ de, Valezi AC. Accuracy of magnetic resonance in identifying traumatic intraarticular knee lesions. Clinics 2005; 60(6): 445-50.

3. Keith Winters, Russell Tregonning. Reliability of magnetic resonance imaging for traumatic injury of the knee. NZMJ [serial online] 2004 Feb [cited 2005 Feb 11]; 118 (1): [1209 screen]. Available from: URL: http://www.nzma.org.nz/journal/118-1209/1301/

4. Edwin H G Oei, Jeroen J Nikken, Antonia C M Verstijnen, Abida Z Ginai, M G Myriam Hunink. MR Imaging of the Menisci and Cruciate Ligaments: A Systematic Review. Radiology 2003; 226: 837-848.

5. Edwin H G Oei, Abida Z Ginai, M G Myriam Hunink. MRI for Traumatic Knee Injury: A Review. Semin Ultrasound CT MRI 2007; 28: 141-157.

6. Singh JP, Garg L, Shrimali R, Setia V, Gupta V. MR Imaging of knee with arthroscopic correlation in twisting injuries. Indian J Radiol Imaging 2004; 14: 33-40.

7. David K B Li, Mark E Adams, J Pat McConkey. Magnetic Resonance Imaging of Ligaments and Menisci of the Knee. Radiol Clin North Am 1986; 24(2): 209-227.

8. S Bryan, G Weatherburn, H Bungay, C Hatrick, C Salas, D Parry, et al. The role of MRI in the diagnosis of knee injuriesHealth Technology Assessment 2001; 5 (27): 11-17.

9. Jill E Langer, Susan J F Meyer, Murray K Dalinka. Imaging of the knee. Radiol Clin North Am1990; 28(5): $975-990$.

10. Douglas P. Bell, J. David Googe, Jason T.Moss, Justin Q. Ly, Barry J. Greer, Annette M. Stapp, et al. Magnetic Resonance Imaging of the Collateral Ligaments and the Anatomic Quadrants of the Knee. Radiol clin N Am 2007; 45: 983-1003.

11. David A. Turner, Chadwick C. Prodromos, Jerry P. Petasnick, John W. Clark. Acute Injury of the Ligaments of the Knee: Magnetic Resonance imaging. Radiology 1985; 154: 717-722.

12. Lee JK, Yao L, Phelps CT, Wirth CR, Czajka J, Lozman J. Anterior cruciate ligament tears: MR

13. imaging compared with arthroscopy and clinical tests. Radiology 1988; 166:861-864.

14. Barronian,A.D., Zoltan and K.A.Bucon. Magnetic resonance imaging of the knee: Correlation with arthroscopy. Arthroscopy 1989; 5:187-191.

15. Heron CW, Calvert PT. Three-dimensional gradient-echo MR imaging of the knee: comparison with arthroscopy in 100 patients. Radiology. 1992;183(3):839-844

16. Tung G A, Davis L M ,Wiggins M E .Tears of the Anterior cruciate ligament: Primary and secondary signs at Mr inaging. Radiology 1993;188;661-667

17. Raunest J, Hotzinger H, Burrig KF. Magnetic resonance imaging (MRI) and arthroscopy in the detection of meniscal degenerations correlation of arthroscopy and MRI with histology findings. Arthroscopy 1994; 10(6):634-640.

18. Mackenzie R, Dixon AK, Keene GS, Hollingworth W, Lomashritte DJ, Villar RN. Magnetic resonance imaging of the knee: assessment of effectiveness. Clin Radiol.1996; 51(4): 245-250.

19. Carmichael I W, Macleod A M.MRI can prevent unnecessary arthroscopy: Journal of Bone and Joint Surgery 1997(4): 624-625

20. Rappeport $\mathrm{ED}^{1}$, Wieslander SB, Stephensen S, Lausten GS, Thomsen HS.Acta Orthop Scand. MRI preferable to diagnostic arthroscopy in knee joint injuries. A double-blind comparison of 47 patients. 1997 Jun;68(3):277-81.

21. Cheung L P , Li K C, Hollett Bergman: Meniscal tears of knee, Accuracy of detection with fast spin Echo MR Imaging and arthroscopic correlation in 293 patients.Radiology 1997.203(2) 508-512 
22. Schultz $\mathrm{T}$, Weilburg et al.Radiology Order entry with decision support-Initial clinical experience.J AM Coll Radiology.2006:3(10)799-806

23. Maurer E J et al,Kaplan P A, Dussault R G et acutely injured knee: Effect of MR imaging in diagnostic and therapeutic decisions.Radiology 1997: 204: 799-805

24. 23.Bui- Mansfield L T, Youngberg R A, Warmer W et al- Potential cost saving of MRI obtained before arthroscopy of knee: Evaluation of 50 consecutive patients: AJR Am J Roentgenol 1997; 168:913-918

25. Stanitski C L Correlation of arthroscopy and clinical examination with MRI findings of injured knees in children and adolescents. AM J Sports Med 1998:26-2

26. Kreitner KF, Runkel M, Herrig A, Regentrop HJ, Grebe P. MRI of knee ligaments: error analysis with reference to meniscus and anterior cruciate ligaments in an arthroscopic controlled patient cohort. Rofo. 1998;169(2):157-162

27. Munk B, Madsen F, Lundorf E, Staunstrup H, Schmidt SA, Bolvig L, Hellfritzch MB, Jensen J (1998) Clinical magnetic resonance imaging and arthroscopic findings in knees: a comparative prospective study of meniscus anterior cruciate ligament and cartilage lesions. Arthroscopy 14(2):171-175

28. Munshi M, Davidson M, MacDonald PB, et al. The efficacy of magnetic resonance imaging in acute knee injuries. Clin J Sport Med. 2000;10:34-39.

29. Zairul-Nizam $Z^{1}$, Hyzan MY, Gobinder S, Razak MA. The role of preoperative magnetic resonance imaging in internal derangement of the knee. Med J Malaysia. 2000 Dec;55(4):433-8.

30. D S Shetty, B N Lakhkar, G K Krishna, Magnetic resonance imaging in pathologic conditions of knee. Indian journal of radiology and imaging,2002, Volume : 12 ; Page : 375-381

31. Vincken PW, ter Braak BP, van Erkel AR, Bloem RM, van Luijt PA, Coene LN, et al. Only MR can safely exclude patients from arthroscopy. Skeletal Radiol 2002;38:977-82

32. Major NM, Beard LN, Jr, Helms CA. Accuracy of MR imaging of the knee in adolescents. AJR Am J Roentgenol.2003;180(1):17-19. doi: 10.2214/ajr.180.1.1800017.

33. Bryan Stirling . Magnetic resonance imaging for investigation of the knee joint: a clinical and economic evaluation. Int $\mathrm{J}$ Technol Assess Health Care. 2004;20(2):222-229.

34. Chang CY, Wu HT, Huang TF, et al. Imaging evaluation of meniscal injury of the knee joint. A comparative MR imaging and arthroscopic study. Clin Imaging 2004; 28: 372-37

35. Thomas Magee ${ }^{1}$, Marc Shapiro and David William. Prevalence of Meniscal Radial Tears of the Knee Revealed by MRI After Surgery April 2004, Vol 182

36. Kolman BH, Daffner RH, Sciulli RL, Soehnlen MW. Correlation of joint fluid and internal derangement on knee MRI. Skeletal Radiol. 2004;33(2):91-95.

37. Kocabey Y, Tetik O, Isbell W, Atay O, Johnson D. The value of clinical examination versus magnetic resonance imaging in the diagnosis of meniscal tears and anterior cruciate ligament rupture. Arthroscopy. 2004;20:696-700.

38. Jee W H, Maccauley T R, Kim J M et al, Meniscal tear configurations. Categorisation with MR Imaging American Journal of Radiology 2004.180;93-97

39. Luhmann SJ, Schootman M, Gordon JE, Wright RW.Magnetic resonance imaging of the knee in children and adolescents. Its role in clinical decision-making. J Bone Joint Surg Am. 2005;87:497-502

40. Esmaili Jah AA, Keyhani S, Zarei R, et al. Accuracy of MRI in comparison with clinical and arthroscopic findings in ligamentous and meniscal injuries of the knee. Acta Orthop Belg. 2005;71:189-196.

41. Carlos Eduardo Sanches Vaz, Olavo Pires de Camargo, Paulo José de Santana,

42. and Antonio Carlos Valezi. Clinics 2005;60(6):445-50: Accuracy of Magnetic Resonance in Identifying traumatic intraarticular knee Lesions

43. Winters K, Tregonning R. Reliability of magnetic resonance imaging of the traumatic knee determined by arthroscopy. N Z Med J. 2005;118(1209):U1301.

44. Mahmoud Karimi-Mobarake, Hamid Barani-Baravati. The Accuracy of Magnetic Imaging Compared with Arthroscopic Finding in Intra-articular Traumatic Knee Injury.Journal Applied Sciences 2005; 5(4): 686-688.

45. Patrice W.J. Vincken, Bert P.M. ter Braak,Arian R.van Erkell,Theo P.W.de Rooy, Walter M.C.Mallens,Wendy Post, et al. Effectiveness of MR Imaging in Selection of Patients for Arthroscopy of the Knee. Radiology 2006 223:739-746.

46. R. Richard Ramnath, Magnetic Resonance Imaging Clinics of North America, Vol 14 No 1, P27, Feb 2006 Br Med Bull. 2007;84:5-23

47. Crawford $\mathrm{R}^{1}$, Walley G, Bridgman S, Maffulli $\mathrm{N}$ Magnetic resonance imaging versus arthroscopy in the diagnosis of knee pathology, concentrating on meniscal lesions and ACL tears: a systematic review. Br Med Bull. 2007;84:5-23.

48. B. R. Mohan and Harminder S. Gosal. Reliability of clinical diagnosis in meniscal tears. Int Orthop Feb 2007:31(1) 5780 
49. Ruth Crawford, Gayle Walley, Stephen Bridgman, Nicola Maffulli. Magnetic resonance imaging versus arthroscopy in the diagnosis of knee pathology, concentrating on meniscal lesions and ACL tears: a systematic review. British Medical Bulletin 2007; 84: 5-23.

50. TR Madhusudhan1*, TM Kumar2, SS Bastawrous3 and A Sinh. Clinical examination, MRI and arthroscopy in meniscal and ligamentous knee Injuries - a prospective study Journal of Orthopaedic Surgery and Research 2008, 3:19

51. Naranje S, Mittal R et al Arthroscopic and magnetic resonance imaging evaluation of meniscus lesions in the chronic anterior cruciate ligament-deficient knee. Department of Orthopaedics, All India Institute of Medical Sciences, New Delhi, India. 2000 Dec; 103(12):1079-85.

52. So Yeon Lee ${ }^{1}$, Won-Hee Jee ${ }^{1}$ and Jung-Man Kim ${ }^{2}$ Radial Tear of the Medial Meniscal Root: Reliability and Accuracy of MRI for Diagnosis. American journal of roentgenology July 2008, Volume 191, Number 1

53. Vassilios S Nikolaou* et al MRI efficacy in diagnosing internal lesions of the knee: a retrospective analysis .Journal of Trauma Management \& Outcomes 2008.

54. Oei $\mathrm{EH}^{1}$, Nikken JJ, Ginai AZ, Krestin GP, Verhaar JA, van Vugt AB, Hunink MG. Costs and effectiveness of a brief MRI examination of patients with acute knee injury. Eur Radiol. 2009 Feb;19(2):409-18.

55. Schurz M,Erdoes J.T., Petras N. The value of clinical examination and MRI versus intraoperative findings in the diagnosis of meniscal tear. Scripta Medica (Brno)-81(1) :3-12 April 2008.

56. Jyrki Halinen, Mika Koivikko, and Eero Hirvensalo The efficacy of magnetic resonance imaging in acute multi-ligament injuries. Int Orthop dec 2009: 33(6) 1733-1738

57. Noha H. Behairy, Mohsen A. Dorgham, and Sherif A. Khaled Accuracy of routine magnetic resonance imaging in meniscal and ligamentous injuries of the knee: comparison with arthroscopy. Int Orthop Aug 2009 :33(4) 963967

58. F. Rayan, Sachin Bhonsle, and Divyang D. Shukla. Clinical, MRI, and arthroscopic correlation in meniscal and anterior cruciate ligament injuries Feb 2009;33(1): 129-132

59. Asif Rahman, Muhammad Nafees, Muhammad Hamid Akram, Atif Hussain Andrabi, Muhammad Zahid. Diagnostic accuracy of magnetic resonance imaging in Meniscal injuries of knee joint and its role in selection of patients for arthroscopy. Radiology Department, Military Hospital Rawalpindi,. J Ayub Med Coll Abbottabad 2010;22(4)

60. Sharma UK, Shrestha BK, Rijal S,Bijukachhe B, Barakoti R, Banskota B, Pradhan I, Banskota AK. Clinical, MRI and arthoscopic correlation in internal derrangement of knee. Kathmandu Univ Med J 2011;35(3):174-8.

61. Julian Dutka, Michał Skowronek, and Łukasz Dutka. Arthroscopic verification of objectivity of the orthopaedic examination and magnetic resonance imaging in intra-articular knee injury. Retrospective study Wideochir Inne Tech MAlo March 2012 : 7(1) 13-18

62. 60. Harish S Lokannavar, Xiaochun Yang, Harsha Guduru. Arthroscopic and Low-Field MRI (0.25 T) Evaluation of Meniscus and Ligaments of Painful Knee. J Clin Imaging Sci 2012, 2:24

63. S Gupta, R Sharma, A Sachar, Y Saini, N Saini. Comparison Of Clinical Examination, MRI And Arthroscopy In Knee Injuries. The Internet Journal of Orthopedic Surgery. 2012 Volume 19 Number 3

64. Schub DL ${ }^{1}$, Altahawi F, F Meisel A, Winalski C, Parker RD, M Saluan P. Accuracy of 3-Tesla magnetic resonance imaging for the diagnosis of intra-articular knee injuries in children and teenagers. J Pediatr Orthop. 2012 Dec;32(8):7659.

65. Gupta Kamini, Guleria Munish, Sandhu Parambir, Galhotra Ritu. Correlation of clinical, MRI and arthroscopic findings in diagnosing meniscus and ligament injuries at knee joint: A prospective study. Journal of orthopaedics and allied scienxces. Volume 1.2013

66. Subhash R Puri, Samar K Biswas, Anil Salgia, Sahil Sanghi, Tushar Aggarwal, Pranav Patel . Study of correlation between clinical, magnetic resonance imaging, and arthroscopic findings in meniscal and anterior cruciate ligament injuries. International Research Journal of Medical Sciences . ISSN 2320 -7353

67. Adil Ismail Nasir. The Role of Magnetic Resonance Imaging in the Knee Joint Injuries. International Science Congress Association 1 Department of Radiology Techniques, Health and Medical Technology - Baghdad Vol. 1(5), 1-7, June (2013) Int. Res. J. Medical Sci.

68. Claus Muhle, Joong Mo Ahn, and Constanze Dieke. Diagnosis of ACL and meniscal injuries: MR imaging of knee flexion versus extension compared to arthroscopy Springerplus 2013: 2;13

69. Kwanseop Lee et al;Anterior cruciate ligament tear-MR based diagnosis in paediatric population,Radiology 1999;213:697-704 